\title{
ON STANDARD $L$-FUNCTIONS ATTACHED TO AUTOMORPHIC FORMS ON DEFINITE ORTHOGONAL GROUPS
}

\author{
ATSUSHI MURASE AND TAKASHI SUGANO
}

\begin{abstract}
We show an explicit functional equation of the standard $L$-function associated with an automorphic form on a definite orthogonal group over a totally real algebraic number field. This is a completion and a generalization of our previous paper, in which we constructed standard $L$-functions by using Rankin-Selberg convolution and the theory of Shintani functions under certain technical conditions. In this article we remove these conditions. Furthermore we show that the $L$-function of $f$ has a pole at $s=m / 2$ if and only if $f$ is a constant function.
\end{abstract}

\section{Introduction}

The purpose of this paper is to prove a meromorphic continuation and a functional equation of the standard $L$-function attached to an auotomorphic form on a definite orthogonal group. In our previous paper [4], we have proposed an approach to construct standard $L$-functions associated with automorphic forms on classical groups. In particular, we proved an explicit functional equation of the standard $L$-function in the case of definite orthogonal groups over $\mathbf{Q}$ under certain conditions. In this paper, removing those technical conditions, we obtain a satisfactory result for the functional equation of the standard $L$-function.

To be more precise, let $k$ be a totally real algebraic number field with maximal order $\mathfrak{o}_{k}$. Let $S \in M_{m}\left(\mathfrak{o}_{k}\right)$ be an even integral (totally) positive definite symmetric matrix of rank $m \geq 2$ and assume that $\mathfrak{o}_{k}^{m}$ is a maximal $\mathfrak{o}_{k}$-integral lattice with respect to $S$. We denote by $G$ the orthogonal group of $S$. For each nonarchimedean place $\mathfrak{p}$, let $K_{\mathfrak{p}}^{*}=\left\{g \in G_{\mathfrak{p}} \mid(g-1) S^{-1} \in\right.$ $\left.M_{m}\left(\mathfrak{o}_{k, \mathfrak{p}}\right)\right\}$, where $G_{\mathfrak{p}}$ is the $\mathfrak{p}$-adic completion of $G$. Clearly $K_{\mathfrak{p}}^{*}$ is a normal subgroup of a maximal open compact subgroup $K_{\mathfrak{p}}=G_{\mathfrak{p}} \cap G L_{m}\left(\mathfrak{o}_{k, \mathfrak{p}}\right)$. We consider the space $\mathfrak{S}\left(K_{f}^{*}\right)$ of left $G_{k}$ and right $G_{\infty} \prod_{\mathfrak{p}<\infty} K_{\mathfrak{p}}^{*}$ invariant functions on the adelized group $G_{A}$ of $G$, where $G_{\infty}$ means the direct product of

Received February 25, 1995. 
$G_{v}$ over all archimedean places $v$. Then the Hecke algebra $\mathcal{H}_{\mathfrak{p}}=\mathcal{H}\left(G_{\mathfrak{p}}, K_{\mathfrak{p}}^{*}\right)$ of the pair $\left(G_{\mathfrak{p}}, K_{\mathfrak{p}}^{*}\right)$ acts on the space $\mathfrak{S}\left(K_{f}^{*}\right)$ naturally. Notice that $\mathcal{H}_{\mathfrak{p}}$ is not commutative in general. We say that $f \in \mathfrak{S}\left(K_{f}^{*}\right)$ is a Hecke eigenform if $f$ is a simultaneous eigenfunction of $\mathcal{H}_{\mathfrak{p}}^{+}$, the center of $\mathcal{H}_{\mathfrak{p}}$, for all $\mathfrak{p}$. For a Hecke eigenform $f$, we denote the local standard $L$-function by $L_{\mathfrak{p}}(f ; s)$, which is normalized in (1.16). At the archimedean places, we introduce the gamma factors $L_{\infty}(f ; s)$ as in (4.3). Our main theorem is as follows.

THEOREM. Let $f \in \mathfrak{S}\left(K_{f}^{*}\right)$ be a simultaneous eigenfunction of $\mathcal{H}_{\mathfrak{p}}^{+}$ for all $\mathfrak{p}$. Then the (completed) standard L-function $\xi(f ; s)=L_{\infty}(f ; s)$ $\prod_{p<\infty} L_{p}(f ; s)$ is continued meromorphically to the whole s-plane and invariant under the transformation $s \mapsto 1-s$. It is holomorphic except for possible simple poles at $s=m / 2-k \quad(0 \leq k \leq m-1, k \in \mathbf{Z})$. Furthermore, $\xi(f ; s)$ has a simple pole at $s=m / 2$ if and only if $f$ is a constant function.

We prove the theorem by an induction on $m$. Let $T \in M_{m+1}\left(\mathfrak{o}_{k}\right)$ be an even integral positive definite symmetric matrix and $S$ the upper left $m \times m$ block of $T$. We assume that $\mathfrak{o}_{k}^{m+1}$ (resp. $\mathfrak{o}_{k}^{m}$ ) is a maximal lattice with respect to $T$ (resp. $S$ ). We embed $G$ into the orthogonal group $H=O(T)$ in a natural manner. Let $F$ (resp. $f$ ) be a right $H_{\infty} \prod_{\mathfrak{p}} U_{\mathfrak{p}}\left(\right.$ resp. $\left.G_{\infty} \prod_{\mathfrak{p}} K_{\mathfrak{p}}\right)$ invariant automorphic form on $H_{A}$ (resp. $G_{A}$ ). We assume that $F$ (resp. $f)$ is a simultaneous eigenfunction of the Hecke algebra of the pair $\left(H_{\mathfrak{p}}, U_{\mathfrak{p}}\right)$ (resp. $\left.\left(G_{\mathfrak{p}}, K_{\mathfrak{p}}\right)\right)$ for all nonarchimedean place p. Under the condition

$$
K_{\mathfrak{p}} \subset U_{\mathfrak{p}}:=H_{\mathfrak{p}} \cap G L_{m+1}\left(\mathfrak{o}_{\mathfrak{p}}\right) \quad \text { for } \quad \forall_{\mathfrak{p}}<\infty,
$$

the main result of [4] asserts that the identity

$$
\int_{H_{k} \backslash H_{A}} F(h) E^{*}(h, f ; s-1 / 2) d h=c<F, \bar{f}>_{G} \cdot \xi(F ; s)
$$

holds. Here $E^{*}$ stands for a normalized Eisenstein series on the orthogonal group $O\left(S_{1}\right)$ of degree $m+2$ and $\langle F, \bar{f}\rangle_{G}$ means the Petersson inner product. Since the analytic continuation and the functional equation of $E^{*}(h, f ; s)$ is obtained from those of $\xi(f ; s)$, our theorem is valid for $F$ if $<F, \bar{f}>_{G} \neq 0$.

To complete the induction argument, we need to remove the condition $[*]$, which is not always satisfied, and to take an $f$ such that $\langle F, \bar{f}\rangle_{G} \neq$ 
0 . This is why we introduce subgroups $K_{\mathfrak{p}}^{*}$ and consider right $G_{\infty} \prod_{\mathfrak{p}} K_{\mathfrak{p}}^{*}$ invariant automorphic forms. Actually, the inclusion

$$
K_{\mathfrak{p}}^{*} \subset U_{\mathfrak{p}}^{*}:=\left\{u \in U_{\mathfrak{p}}^{*} \mid(u-1) T^{-1} \in M_{m+1}\left(\mathfrak{o}_{k, \mathfrak{p}}\right)\right\}
$$

is always satisfied. Moreover, the identity (0.1) holds for any Hecke eigenforms $F \in \mathfrak{S}\left(U_{f}^{*}\right)$ and $f \in \mathfrak{S}\left(K_{f}^{*}\right)$.

We now give a brief account of the paper. In $\S 1$, we determine the structures of $\mathcal{H}_{\mathfrak{p}}$ and its subalgebra $\mathcal{H}_{\mathfrak{p}}^{+}$respectively (Theorem 1.3) and describe the Satake isomorphisms (Theorem 1.8). After introducing local standard $L$-functions, we calculate certain integrals needed in $\S 4$. In $\S 2$, we define certain norm functions on $G$, which appear in an integral expression of local $L$-functions (Theorem 2.10). We recall the definition of local Shintani functions and prove an integral formula for them, which follows from several properties of norm functions. The object of the next section is to prove a crucial fact about arithmetic of quadratic forms (Theorem 3.3), which enables us to take an $f$ such that $\langle F, \bar{f}\rangle_{G} \neq 0$. Namely we show that, for any even integral, maximal, positive definite symmetric matrix $T$ of rank $m+1$, there exists an element $\gamma$ in $S L_{m+1}\left(\mathfrak{o}_{k}\right)$ such that the upper left $m \times m$ bolck of $T[\gamma]$ satisfies the maximality condition. In $\S 4$, our main result is proved by combining the result of Eisenstein series (Proposition 4.3) and the basic identity (Theorem 4.4) together with local results proved in the preceding sections.

Notation. For a ring $R$, we denote by $\operatorname{Sym}_{m}(R)$ the set of symmetric matrices of degree $m$ with entries in $R$. For $S \in \operatorname{Sym}_{m}(R)$ and $X, Y \in$ $M_{m, n}(R)$, we put $S(X, Y)={ }^{t} X S Y$ and $S[X]=S(X, X)$. For an algebraic group $G$ defined over an algebraic number field $k$ and a place $v$ of $k$, we denote $G_{v}$ (resp. $G_{A}$ ) the completion of $G$ at $v$ (resp. the adelization of $G)$.

\section{$\S 1$. Hecke algebra}

\subsection{Preliminaries}

In this subsection we recall several facts on quadratic forms and orthogonal groups over local fields. Let $k$ be a nonarchimedean local field with characteristic different from 2 . We denote by $\mathfrak{o}$ the maximal order of $k$, by $\mathfrak{p}=(p)$ the maximal ideal of $\mathfrak{o}$ and by $q$ the order of the residual field. We normalize the valuation ||$=||_{\mathfrak{p}}$ of $k$ so that $|p|=q^{-1}$ and 
put $\operatorname{ord}_{\mathfrak{p}}(x)=-\log _{q}(|x|)$ for $x \in k^{\times}$. A non-degenerate symmetric ma$\operatorname{trix} S=\left(s_{i j}\right) \in M_{m}(k)$ is said to be even integral if $s_{i j} \in \mathfrak{o}$ and $s_{i i} \in 2 \mathfrak{o}$ for $1 \leq i, j \leq m$. We say that $S$ is maximal if $L=\mathfrak{o}^{m}$ is a maximal o-integral lattice with respect to $S$; namely, there is no integral matrix $g \in M_{m}(\mathfrak{o}) \cap G L_{m}(k)-G L_{m}(\mathfrak{o})$ such that $S\left[g^{-1}\right]$ is also even integral. We denote by $L^{*}=S^{-1} L$ the dual lattice of $L$ with respect to $S$. From now on we assume that $S$ is maximal. Taking a suitable $\mathfrak{o}$-basis of $L$, we may assume

$$
\begin{aligned}
& S=S_{\nu}=\left[\begin{array}{ccc} 
& & J_{\nu} \\
& S_{0} & \\
J_{\nu} & &
\end{array}\right], \\
& J_{\nu}=\left[\begin{array}{lll} 
& . & 1 \\
1 & &
\end{array}\right] \in G L_{\nu}(k) \quad(1 \text { appears } \nu \text { times }),
\end{aligned}
$$

where $\nu=\nu(S)=\nu_{\mathfrak{p}}(S)$ is the Witt index of $S$ over $k$ and $S_{0}$ is an anisotropic maximal even integral matrix of size $n_{0}: n_{0}=n_{0}(S)=n_{0, \mathfrak{p}}(S)$ $=m-2 \nu$. It is well-known that $0 \leq n_{0} \leq 4$. From the maximality condition, $L^{\prime}=\left\{x \in L^{*} \mid S[x] / 2 \in \mathfrak{p}^{-1}\right\}$ forms a lattice and $L^{\prime} / L$ admits a structure of a vector space over $\mathfrak{o} / \mathfrak{p}$ equipped with a non-degenerate quadratic form $\frac{1}{2} p S[x]$. We denote its dimension by $\partial=\partial(S)=\partial_{\mathfrak{p}}(S)$. For the sake of convenience, we quote the list of maximal anisotropic even integral symmetric matrices following Eichler [1, Satz 9.7].

(1.2) List of maximal anisotropic even integral symmetric matrices

\begin{tabular}{|c|c|c|c|r|}
\hline$n_{0}$ & $\partial$ & $S_{0}$ & $\operatorname{ord}_{\mathfrak{p}}\left(\operatorname{det} S_{0}\right)$ & $\chi_{\mathrm{MH}}\left(S_{0}\right)$ \\
\hline 0 & 0 & & 0 & 1 \\
\hline 1 & 0 & $2 s$ & $e$ & 1 \\
\hline 1 & 1 & $2 p s$ & $e+1$ & 1 \\
\hline 2 & 0 & $s S_{k_{0} / k}$ & 0 & 1 \\
\hline 2 & 1 & $s S_{k_{1} / k}$ & $2 f(1 \leq f \leq e)$ or $2 e+1$ & \pm 1 \\
\hline 2 & 2 & $p s S_{k_{0} / k}$ & 2 & -1 \\
\hline 3 & 1 & $2 p s \oplus s^{\prime} S_{k_{0} / k}$ & $e+1$ & -1 \\
\hline 3 & 2 & $2 s \oplus p s^{\prime} S_{k_{0} / k}$ & $e+2$ & -1 \\
\hline 4 & 2 & $s S_{k_{0} / k} \oplus p s^{\prime} S_{k_{0} / k}$ & 2 & -1 \\
\hline
\end{tabular}


Here $e=\operatorname{ord}_{\mathfrak{p}}(2), \chi_{\mathrm{MH}}$ stands for the Minkowski-Hasse character and $s, s^{\prime} \in$ $\mathfrak{o}^{\times}$. For a quadratic extension $k^{\prime}$ over $k$, identifying its maximal order with $\mathfrak{o}^{2}$, we put $S_{k^{\prime} / k}(X, Y)=\operatorname{Tr}_{k^{\prime} / k}(\bar{X} Y)$. In the above list, $k_{0}$ (resp. $\left.k_{1}\right)$ is the unique unramified quadratic extension (resp. a ramified extension ) of $k$.

Let $G$ be the orthogonal group of $S$ and put

$$
K=G \cap G L_{m}(\mathfrak{o}) \quad \triangleright \quad K^{*}=\left\{g \in K \mid(g-1) L^{*} \subset L\right\}
$$

First we consider the structure of the quotient group $E=K / K^{*}$. We denote by $\operatorname{Aut}\left(L^{*} / L\right)$ the set of o-module isomorphisms $\sigma$ of $L^{*} / L$ such that $\frac{1}{2} S[\sigma(x)] \equiv \frac{1}{2} S[x] \quad(\bmod \mathfrak{o})$ for any $x \in L^{*}$. Each $u \in K$ defines the element $\varphi(u) \in \operatorname{Aut}\left(L^{*} / L\right)$ by $\varphi(u)(x)=u x\left(x \in L^{*}\right)$.

Proposition 1.1. The mapping $\varphi$ is an isomorphism beteween $K / K^{*}$ and $\operatorname{Aut}\left(L^{*} / L\right)$. Furthermore

$$
\operatorname{Aut}\left(L^{*} / L\right) \cong\left\{\begin{array}{cl}
\{1\} & \text { if } \partial=0 \\
\mathbf{Z} / 2 \mathbf{Z} & \text { if } \partial=1 \\
D_{q+1} & \text { if } \partial=2
\end{array}\right.
$$

where $D_{q+1}$ is the dihedral group of order $2(q+1)$.

Proof. It is obvious that $\varphi$ is an injective homomorphism. To see the surjectivity, we may assume that $S$ is anisotropic because of (1.1). We can check the surjectivity case-by-case following the list (1.2). For example, we consider the case $\left(n_{0}, \partial\right)=(2,2)$. Let $k_{0}$ (resp. $\left.\mathfrak{o}_{0}\right)$ be the unramified quadratic extension field over $k$ (resp. the maximal order of $k_{0}$ ). We set $S=p \cdot S_{k_{0} / k}=p \cdot\left[\begin{array}{cc}2 & b \\ b & 2 c\end{array}\right]$, where $\{1, \omega\}$ is an $\mathfrak{o}$-basis of $\mathfrak{o}_{0}$ and $\omega^{2}-b \omega+$ $c=0$ is the minimal polynomial of $\omega$ over $k$. Take any $\sigma \in \operatorname{Aut}\left(L^{*} / L\right)$ and put $\sigma\left(\left[\begin{array}{l}1 \\ 0\end{array}\right] p^{-1}\right) \equiv\left[\begin{array}{l}x \\ y\end{array}\right] p^{-1}(\bmod L)$. Since $\mathrm{N}_{k_{0} / k}\left(\mathfrak{o}_{0}^{\times}\right)=\mathfrak{o}^{\times}$, we may suppose that $x+\omega y$ is in $\mathfrak{o}_{0}^{(1)}=\left\{\alpha \in \mathfrak{o}_{0} \mid \mathrm{N}_{k_{0} / k}(\alpha)=1\right\}$. Then $g_{x+\omega y}=\left[\begin{array}{cc}x & -c y \\ y & x+b y\end{array}\right]$ is in $K$ and $\sigma^{\prime}=\varphi\left(g_{x+\omega y}\right)^{-1} \sigma$ stabilizes $\left[\begin{array}{l}1 \\ 0\end{array}\right] p^{-1}$ modulo $L$. It is easily seen that $\sigma^{\prime}\left(\left[\begin{array}{l}0 \\ 1\end{array}\right] p^{-1}\right) \equiv\left[\begin{array}{l}0 \\ 1\end{array}\right] p^{-1}$ or $\left[\begin{array}{c}b \\ -1\end{array}\right] p^{-1}$ $(\bmod L)$, namely $\sigma^{\prime}=\varphi(1)$ or $\varphi\left(\left[\begin{array}{cc}1 & b \\ 0 & -1\end{array}\right]\right)$. Therefore we know that $\varphi$ is 
surjective. Moreover from the above argument, $\operatorname{Aut}\left(L^{*} / L\right)$ is isomorphic to the semidirect product of $\mathfrak{o}_{0}^{(1)} /\left(\mathfrak{o}_{0}^{(1)} \cap\left(1+p \mathfrak{o}_{0}\right)\right) \cong \mathbf{Z} /(q+1) \mathbf{Z}$ and the Galois group of $k_{0} / k$. The other cases are treated similarly.

From now on we suppose that $S$ is of the form (1.1). When we need to emphasize the Witt index $\nu$, we write $\nu$ as a suffix; $G=G_{\nu}, K=K_{\nu}, L=L_{\nu}$ etc. For $a_{1}, \ldots, a_{\nu} \in k^{\times}$and $u \in G_{0}=K_{0}$, we put

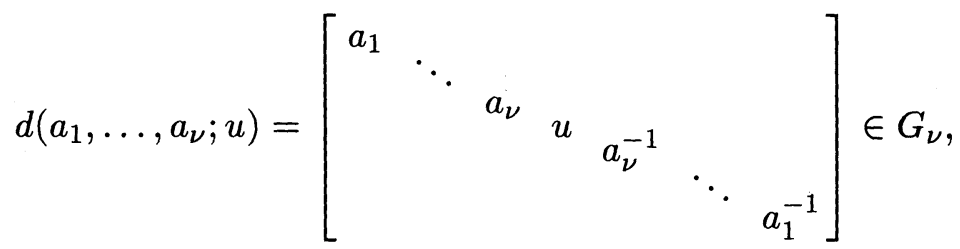

$$
\begin{aligned}
& H_{\nu}=\left\{g=d\left(a_{1}, \ldots, a_{\nu} ; u\right) \mid a_{1}, \ldots, a_{\nu} \in k^{\times}, u \in K_{0}\right\}, \\
& A_{\nu}=\left\{g=d\left(a_{1}, \ldots, a_{\nu} ; 1_{n_{0}}\right) \mid a_{1}, \ldots, a_{\nu} \in k^{\times}\right\} \quad \text { and } \\
& N_{\nu}=\left\{g=\left[\begin{array}{ccc}
n_{1} & * & * \\
& 1_{n_{0}} & * \\
& & n_{1}^{\prime}
\end{array}\right] \mid n_{1}=\left[\begin{array}{ccc}
1 & * & * \\
& \ddots & * \\
& & 1
\end{array}\right]\right. \\
& \left.\in M_{\nu}(k), n_{1}^{\prime}=J_{\nu}^{-1 t} n_{1}^{-1} J_{\nu}\right\} \text {. }
\end{aligned}
$$

Identifying $u \in K_{0}$ with $d(1, \ldots, 1 ; u) \in K_{\nu}$, we have $K_{\nu}=K_{0} K_{\nu}^{*}$ because of Proposition 1.1. For an $n$-tuple $\mathbf{r}=\left(r_{1}, \ldots, r_{\nu}\right) \in \mathbf{Z}^{\nu}$ and $u \in K_{0}$, we set

$$
\pi_{\mathbf{r}, u}=d\left(p^{r_{1}}, \ldots, p^{r_{\nu}} ; u\right) \in H_{\nu} \quad \text { and } \quad \pi_{\mathbf{r}}=\pi_{\mathbf{r}, 1} \in A_{\nu}
$$

From the usual Iwasawa and Cartan decompositions $G_{\nu}=N_{\nu} H_{\nu} K_{\nu}=$ $K_{\nu} H_{\nu} K_{\nu}$, we have

Proposition 1.2. The following Iwasawa and Cartan decompositions hold.

$$
\begin{aligned}
& G_{\nu}=N_{\nu} H_{\nu} K_{\nu}^{*}=\coprod_{\mathbf{r} \in \mathbf{Z}^{\nu}, \varepsilon \in E} N_{\nu} \pi_{\mathbf{r}, \varepsilon} K_{\nu}^{*} \\
& G_{\nu}=K_{\nu}^{*} H_{\nu} K_{\nu}^{*}=\coprod_{\mathbf{r} \in \Lambda_{\nu}, \varepsilon \in E} K_{\nu}^{*} \pi_{\mathbf{r}, \varepsilon} K_{\nu}^{*}
\end{aligned}
$$

where $E=K_{\nu} / K_{\nu}^{*}$ and $\Lambda_{\nu}=\left\{\mathbf{r}=\left(r_{1}, \ldots, r_{\nu}\right) \in \mathbf{Z}^{\nu} \mid r_{1} \geq \cdots \geq r_{\nu} \geq 0\right\}$. 


\subsection{Structure of Hecke algebra}

We denote by $\mathcal{H}=\mathcal{H}_{\nu}=\mathcal{H}\left(G, K^{*}\right)$ the Hecke algebra of the pair $\left(G, K^{*}\right)$. Namely, $\mathcal{H}$ is the set of compactly supported, bi- $K^{*}$-invariant $\mathbf{C}$-valued functions on $G$. Then $\mathcal{H}$ forms a $\mathbf{C}$-algebra by the convolution product:

$$
\left(f_{1} * f_{2}\right)(x)=\int_{G} f_{1}\left(x y^{-1}\right) f_{2}(y) d y \quad\left(f_{1}, f_{2} \in \mathcal{H}_{\nu}\right),
$$

where $d y$ is the Haar measure of $G$ normalized so that the total volume of $K^{*}$ is 1 . For any $g \in G$, we denote by $f_{[g]}$ the characteristic function of $K^{*} g K^{*}$ and we put $f_{\mathbf{r}, \varepsilon}=f_{\left[\pi_{\mathbf{r}, \varepsilon}\right]}$. Proposition 1.2 says that $\left\{f_{\mathbf{r}, \varepsilon} \mid \mathbf{r} \in\right.$ $\left.\Lambda_{\nu}, \varepsilon \in E=K / K^{*}\right\}$ forms a $\mathbf{C}$-basis of $\mathcal{H}$. We introduce a subalgebra of $\mathcal{H}$ by

(1.6) $\mathcal{H}^{+}=\mathcal{H}_{\nu}^{+}=\mathcal{H}^{+}\left(G, K^{*}\right)=\left\{f \in \mathcal{H} \mid f\left(u^{-1} g u\right)=f(g)\right.$ for $\left.{ }^{\forall} u \in K\right\}$.

Our aim in this subsection is to determine the structures of $\mathcal{H}^{+}$and $\mathcal{H}$. Let $B=\mathbf{C}[E]$ be the group algebra of $E=K_{\nu} / K_{\nu}^{*}=K_{0} / K_{0}^{*}$ over $\mathbf{C}$ and $Z(B)$ its center. Let $X_{1}, \ldots, X_{\nu}$ be independent variables over $\mathbf{C}$ and $\mathbf{C}\left[X_{1}^{ \pm}, \ldots, X_{\nu}^{ \pm}\right]$the polynomial ring generated by $X_{1}, X_{1}^{-1}, \ldots, X_{\nu}, X_{\nu}^{-1}$ over $\mathbf{C}$. Then $B\left[X_{1}^{ \pm}, \ldots, X_{\nu}^{ \pm}\right]=B \otimes_{\mathbf{C}} \mathbf{C}\left[X_{1}^{ \pm}, \ldots, X_{\nu}^{ \pm}\right]$forms a $\mathbf{C}$-algebra naturally. We denote by $W_{\nu}$ the group of automorphisms of $B\left[X_{1}^{ \pm}, \ldots, X_{\nu}^{ \pm}\right]$ generated by all permutations of $X_{1}, \ldots, X_{\nu}$ and $\sigma_{i}: X_{i} \rightarrow X_{i}^{-1}, X_{j} \rightarrow$ $X_{j}(j \neq i)$.

For $f \in \mathcal{H}_{\nu}, h \in H_{\nu}, \mathbf{r} \in \mathbf{Z}^{\nu}$ and $\varepsilon \in E$, we put

$$
\Phi_{f}(h)=\delta(h)^{1 / 2} \int_{N_{\nu}} f(h n) d n \quad \text { and } \quad \Phi_{f}(\mathbf{r}, \varepsilon)=\Phi_{f}\left(\pi_{\mathbf{r}, \varepsilon}\right),
$$

where $d n$ is the Haar measure of $N_{\nu}$ normalized so that the volume of $N_{\nu} \cap K_{\nu}^{*}$ is 1 and

$$
\delta(h)=d\left(h n h^{-1}\right) / d n \quad \text { for } \quad h \in H_{\nu} .
$$

THEOREM 1.3. Through the mapping

$$
\begin{aligned}
\Phi: f \longmapsto \Phi_{f}(\mathbf{X})= & \sum_{\dot{\mathbf{r}} \in \mathbf{Z}^{\nu}, \varepsilon \in E} \Phi_{f}(\mathbf{r}, \varepsilon) \varepsilon \mathbf{X}^{\mathbf{r}} \\
& \left(\mathbf{X}^{\mathbf{r}}=X_{1}^{r_{1}} \cdots X_{\nu}^{r_{\nu}} \text { for } \mathbf{r}=\left(r_{1}, \ldots, r_{\nu}\right)\right),
\end{aligned}
$$

the Hecke algebra $\mathcal{H}_{\nu}$ (resp. $\mathcal{H}_{\nu}^{+}$) is isomorphic to the algebra $B\left[X_{1}^{ \pm}, \ldots\right.$, $\left.X_{\nu}^{ \pm}\right]^{W_{\nu}}$ (resp. $Z(B)\left[X_{1}^{ \pm}, \ldots, X_{\nu}^{ \pm}\right]^{W_{\nu}}$ ) of $W_{\nu}$-invariant polynomials. 
Proof. Clearly $\Phi$ is a $\mathbf{C}$-algebra homomorphism of $\mathcal{H}_{\nu}$ into $B\left[X_{1}^{ \pm}, \ldots\right.$, $X_{\nu}^{ \pm}$. To see the injectivity and to determine the image of $\Phi$, we trace the argument in Satake $[5, \S 6]$. For $h=d\left(a_{1}, \ldots, a_{\nu} ; \varepsilon\right) \in H_{\nu}$, we put

$$
D(h)=\prod_{i<j}\left|1-a_{i}^{-1} a_{j}\right|\left|1-a_{i}^{-1} a_{j}^{-1}\right| \prod_{i=1}^{\nu}\left|\operatorname{det}\left(1-\varepsilon^{-1} a_{i}^{-1}\right)\right| .
$$

When $D(h) \neq 0, n \mapsto h^{-1} n^{-1} h n$ gives a bijection from $N_{\nu}$ to itself and $d\left(h^{-1} n^{-1} h n\right)=D(h) d n$. Because of $\delta(h)=\prod_{i=1}^{\nu}\left|a_{i}\right|^{2\left(\nu-i+n_{0} / 2\right)}$, we know that

$$
\begin{aligned}
\Delta(h) & \stackrel{\text { def }}{=} D(h) \delta(h)^{1 / 2} \\
& =\prod_{i<j}\left|a_{i}+a_{i}^{-1}-\left(a_{j}+a_{j}^{-1}\right)\right| \prod_{i=1}^{\nu}\left|\operatorname{det}\left(a_{i}+a_{i}^{-1}-\varepsilon-\varepsilon^{-1}\right)\right|^{1 / 2}
\end{aligned}
$$

is invariant under the Weyl group $W_{\nu}$ and the conjugation by $u \in K_{0}$ : $\varepsilon \mapsto u^{-1} \varepsilon u$. If $D(h) \neq 0$ and $f \in \mathcal{H}_{\nu}^{+}$, then

$$
\int_{A_{\nu} \backslash G_{\nu}} f\left(g^{-1} h g\right) d g=\Delta(h)^{-1} \Phi_{f}(h) \int_{K_{\nu}} d u .
$$

Thus we obtain $\Phi_{f}\left(w \mathbf{r}, u^{-1} \varepsilon u\right)=\Phi_{f}(\mathbf{r}, \varepsilon)\left(f \in \mathcal{H}_{\nu}^{+}, w \in W_{\nu}, u \in K_{0}\right)$ and $\Phi\left(\mathcal{H}_{\nu}^{+}\right) \subset Z(B)\left[X_{1}^{ \pm}, \ldots, X_{\nu}^{ \pm}\right]^{W_{\nu}}$. Let $\left\{C_{1}, \ldots, C_{l}\right\}$ be the set of conjugate classes of $E$ and put $e_{i}=\sum_{\varepsilon \in C_{i}} \varepsilon$. Then it is well-known that $\left\{e_{1}, \ldots, e_{l}\right\}$ forms a $\mathbf{C}$-basis of $Z(B)$. Therefore as a $\mathbf{C}$-basis of $\mathcal{H}_{\nu}^{+}$(resp. $Z(B)\left[\mathbf{X}^{ \pm}\right]^{W_{\nu}}$ ) we can take $\left\{\sum_{\varepsilon \in C_{i}} f_{\mathbf{r}, \varepsilon} \mid \mathbf{r} \in \Lambda_{\nu}, 1 \leq i \leq l\right\}$ (resp. $\left\{e_{i} \sum_{w \in W_{\nu}} \mathbf{X}^{w \mathbf{r}} \mid \mathbf{r} \in \Lambda_{\nu}, 1 \leq\right.$ $i \leq l\}$ ). The representation matrix of $\Phi$ with respect to these bases is upper triangular and non-degenerate. Therefore we get the bijectivity of $\Phi \mid \mathcal{H}_{\nu}^{+}$. Using the fact that $f_{\mathbf{r}, \varepsilon} * f_{\left[\varepsilon^{\prime}\right]}=f_{\mathbf{r}, \varepsilon \varepsilon^{\prime}}$ and $\Phi\left(f_{[\varepsilon]}\right)=\varepsilon$, we similarly obtain the surjectivity of $\Phi$.

Remark 1.4. It follows from Theorem 1.3 that $\mathcal{H}_{\nu}^{+}$is the center of $\mathcal{H}_{\nu}$.

\subsection{Zonal spherical functions}

We say that a function $\omega$ on $G_{\nu}$ is a zonal spherical function on $G_{\nu}$ 
(z.s.f. for brevity), if it satisfies the following conditions.

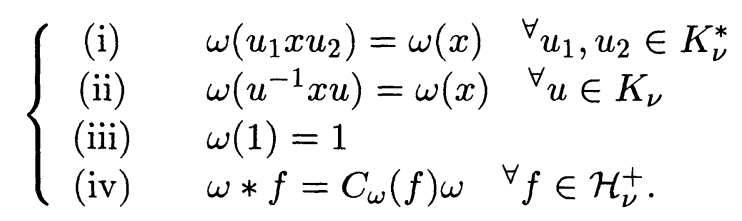

From the definition, $C_{\omega}(f)=\int_{G_{\nu}} \omega(x) f\left(x^{-1}\right) d x$ defines a character of $\mathcal{H}_{\nu}^{+}$ (a $\mathbf{C}$-algebra homomorphism of $\mathcal{H}_{\nu}^{+}$to $\mathbf{C}$ ). As in the usual bi- $K$-invariant case, a z.s.f. $\omega$ is uniquely determined by its character $C_{\omega}$ up to a constant. Indeed, the following assertion holds.

LEMMA 1.5. Let $\varphi$ be a bi-K $K_{\nu}^{*}$-invariant function on $G_{\nu}$. If $\varphi$ satisfies $\varphi\left(u^{-1} x u\right)=\varphi(x)$ for any $u \in E$ and $(\varphi * f)(1)=0$ for any $f \in \mathcal{H}_{\nu}^{+}$, then $\varphi=0$.

Proof. Our proof is quite similar to that in Tamagawa [7, §2]. Take any continuous function $f$ on $G_{\nu}$ with compact support and put

$$
f^{0}(x)=\int_{K_{\nu}^{*}} \int_{K_{\nu}^{*}} f\left(u_{1} x u_{2}\right) d u_{1} d u_{2} \quad \text { and } \quad f^{00}(x)=|E|^{-1} \sum_{u \in E} f^{0}\left(u^{-1} x u\right) .
$$

Clearly $f^{0} \in \mathcal{H}_{\nu}$ and $f^{00} \in \mathcal{H}_{\nu}^{+}$. From our assumption we have

$$
\begin{aligned}
0 & =\int_{G_{\nu}} \varphi\left(y^{-1}\right) f^{00}(y) d y=|E|^{-1} \sum_{u \in E} \int_{G_{\nu}} \varphi\left(y^{-1}\right) f^{0}\left(u^{-1} y u\right) d y \\
& =|E|^{-1} \sum_{u \in E} \int_{G_{\nu}} \varphi\left(u y^{-1} u^{-1}\right) f^{0}(y) d y=\int_{G_{\nu}} \varphi\left(y^{-1}\right) f^{0}(y) d y \\
& =\int_{G_{\nu}} \int_{K_{\nu}^{*}} \int_{K_{\nu}^{*}} \varphi\left(y^{-1}\right) f\left(u_{1} y u_{2}\right) d u_{1} d u_{2} d y=\int_{G_{\nu}} \varphi\left(y^{-1}\right) f(y) d y .
\end{aligned}
$$

Thus we get $\varphi=0$.

Now we construct all zonal spherical functions. Take any irreducible representation $\rho$ of $E$ with underlying vector space $V_{\rho}$ and denote by $\chi_{\rho}$ (resp. $n_{\rho}$ ) the character (resp. the degree) of $\rho$. The same letter $\rho$ is used for the corresponding representation of the group ring $B$. For a $\nu$-tuple 
$\boldsymbol{\lambda}=\left(\lambda_{1}, \ldots, \lambda_{\nu}\right)$ of unramified characters of $k^{\times}$, we define an $\operatorname{End}\left(V_{\rho}\right)$ valued function $\phi_{\boldsymbol{\lambda}, \rho}$ on $G_{\nu}$ by

$$
\begin{aligned}
\phi_{\boldsymbol{\lambda}, \rho}(h n u) & =\rho(\varepsilon) \delta(h)^{1 / 2} \prod_{i=1}^{\nu} \lambda_{i}\left(a_{i}\right) \\
& =\rho(\varepsilon) \prod_{i=1}^{\nu} \lambda_{i}\left(a_{i}\right)\left|a_{i}\right|^{\nu-i+n_{0} / 2} \in \operatorname{End}\left(V_{\rho}\right)
\end{aligned}
$$

where $h=d\left(a_{1}, \ldots, a_{\nu} ; \varepsilon\right) \in H_{\nu}, n \in N_{\nu}$ and $u \in K_{\nu}^{*}$. Clearly

$$
\phi_{\boldsymbol{\lambda}, \rho}\left(h_{0} g u_{0}\right)=\phi_{\boldsymbol{\lambda}, \rho}\left(h_{0}\right) \phi_{\boldsymbol{\lambda}, \rho}(g) \rho\left(u_{0}\right) \quad \text { for } h_{0} \in H_{\nu}, u_{0} \in K_{\nu}
$$

LeMma 1.6. For any $f \in \mathcal{H}_{\nu}$,

$$
\phi_{\boldsymbol{\lambda}, \rho} * f=\phi_{\boldsymbol{\lambda}, \rho} \cdot C_{\boldsymbol{\lambda}, \rho}(f), \quad C_{\boldsymbol{\lambda}, \rho}(f)=\int_{G_{\nu}} \phi_{\boldsymbol{\lambda}, \rho}\left(y^{-1}\right) f(y) d y .
$$

In particular, $C_{\boldsymbol{\lambda}, \rho}(f) \in \mathbf{C}$ for $f \in \mathcal{H}_{\nu}^{+}$.

Proof. For $x=h_{0} n_{0} u_{0}\left(h_{0} \in H_{\nu}, n_{0} \in N_{\nu}, u_{0} \in K_{\nu}^{*}\right)$, we have

$$
\begin{aligned}
\left(\phi_{\boldsymbol{\lambda}, \rho} * f\right)(x) & =\int_{G_{\nu}} \phi_{\boldsymbol{\lambda}, \rho}(y) f\left(y^{-1} h_{0} n_{0}\right) d y=\int_{G_{\nu}} \phi_{\boldsymbol{\lambda}, \rho}\left(h_{0} n_{0} y\right) f\left(y^{-1}\right) d y \\
& =\phi_{\boldsymbol{\lambda}, \rho}\left(h_{0}\right) \int_{G_{\nu}} \phi_{\boldsymbol{\lambda}, \rho}(y) f\left(y^{-1}\right) d y
\end{aligned}
$$

and hence get the first assertion. If $f \in \mathcal{H}_{\nu}^{+}$, for any $u \in E$,

$$
C_{\boldsymbol{\lambda}, \rho}(f)=\int_{G_{\nu}} \phi_{\boldsymbol{\lambda}, \rho}\left(u y u^{-1}\right) f\left(u y^{-1} u^{-1}\right) d y=\rho(u) C_{\boldsymbol{\lambda}, \rho}(f) \rho(u)^{-1}
$$

Since $\rho$ is irreducible, we have $C_{\boldsymbol{\lambda}, \rho}(f) \in \mathbf{C}$.

We define a $\mathbf{C}$-valued function on $G_{\nu}$ by

$$
\omega_{\boldsymbol{\lambda}, \rho}(x)=\frac{1}{n_{\rho}} \int_{K_{\nu}^{*}} \operatorname{tr}\left(\phi_{\boldsymbol{\lambda}, \rho}(v x)\right) d v .
$$

LEMMA 1.7. The function $\omega_{\boldsymbol{\lambda}, \rho}$ is a zonal spherical function corresponding to the character $C_{\boldsymbol{\lambda}, \rho}$. 
Proof. It is clear that $\omega_{\boldsymbol{\lambda}, \rho}$ is bi- $K_{\nu}^{*}$-invariant and $\omega_{\boldsymbol{\lambda}, \rho}(1)=1$. For any $u \in E$ we have

$$
\begin{aligned}
\omega_{\boldsymbol{\lambda}, \rho}\left(u x u^{-1}\right) & =\frac{1}{n_{\rho}} \int_{K_{\nu}^{*}} \operatorname{tr}\left(\phi_{\boldsymbol{\lambda}, \rho}\left(v u x u^{-1}\right)\right) d v=\frac{1}{n_{\rho}} \int_{K_{\nu}^{*}} \operatorname{tr}\left(\phi_{\boldsymbol{\lambda}, \rho}\left(u v^{\prime} x u^{-1}\right)\right) d v^{\prime} \\
& =\frac{1}{n_{\rho}} \int_{K_{\nu}^{*}} \operatorname{tr}\left(\rho(u) \phi_{\boldsymbol{\lambda}, \rho}(v x) \rho(u)^{-1}\right) d v=\omega_{\boldsymbol{\lambda}, \rho}(x) .
\end{aligned}
$$

Finally for any $f \in \mathcal{H}_{\nu}^{+}$, by Lemma 1.6,

$$
\begin{aligned}
\left(\omega_{\boldsymbol{\lambda}, \rho} * f\right)(x) & =\int_{G_{\nu}} \frac{1}{n_{\rho}} \int_{K_{\nu}^{*}} \operatorname{tr}\left(\phi_{\boldsymbol{\lambda}, \rho}(v y)\right) f\left(y^{-1} x\right) d y d v \\
& =\frac{1}{n_{\rho}} \int_{K_{\nu}^{*}} \operatorname{tr}\left\{\int_{G_{\nu}} \phi_{\boldsymbol{\lambda}, \rho}(y) f\left(y^{-1} v x\right) d y\right\} d v \\
& =\frac{1}{n_{\rho}} \int_{K_{\nu}^{*}} \operatorname{tr}\left(\phi_{\boldsymbol{\lambda}, \rho}(v x) C_{\boldsymbol{\lambda}, \rho}(f)\right) d v=C_{\boldsymbol{\lambda}, \rho}(f) \cdot \omega_{\boldsymbol{\lambda}, \rho}(x),
\end{aligned}
$$

and hence our assertion is proved.

Let $\left\{C_{1}, \ldots, C_{l}\right\}$ be the set of conjugate classes of $E$ and $\rho$ be an irreducible representation of $E$. Then

$$
\sum_{u \in C_{i}} u \longmapsto \frac{1}{n_{\rho}} \sum_{u \in C_{i}} \chi_{\rho}(u)
$$

gives a $\mathbf{C}$-algebra homomorphism of $Z(B)$ into $\mathbf{C}$. Conversely every $\mathbf{C}$ algebra homomorphism of $Z(B)$ is obtained in this way. Therefore we get the following theorem.

Theorem 1.8. Any $\mathbf{C}$-algebra homomorphism $\Lambda$ of $\mathcal{H}_{\nu}^{+}$into $\mathbf{C}$ comes from the character corresponding to a zonal spherical function on $G_{\nu}$. In particular, there exists a $\nu$-tuple $\boldsymbol{\lambda}=\left(\lambda_{1}, \ldots, \lambda_{\nu}\right)$ of unramified characters of $k^{\times}$and an irreducible representation $\rho$ of $E$ such that $\Lambda=C_{\boldsymbol{\lambda}, \rho}$, where $\boldsymbol{\lambda}$ (resp. $\rho$ ) is uniquely determined modulo $W_{\nu}$ (resp. up to equivalence). We call $(\boldsymbol{\lambda}, \rho)$ the Satake parameter of $\Lambda$. 


\subsection{Local standard $L$-functions}

From Proposition 1.1, $E$ has a unique cyclic normal subgroup $C$ of index 2 , if $\partial \geq 1$. We denote by $\rho_{1}$ the one dimensional non-trivial representation of $E$ whose restriction to $C$ is trivial. For an irreducible representation $\rho$ of $E$, we define a number $\lambda_{0}=\lambda_{0}(\rho)$ by

$$
\lambda_{0}(\rho)=\left\{\begin{aligned}
1 & \text { if } \rho \text { is trivial } \\
-1 & \text { if } \rho=\rho_{1} \\
0 & \text { otherwise }
\end{aligned}\right.
$$

In particular $\lambda_{0}(\rho)=1$ (resp. $\left.\lambda_{0}(\rho)= \pm 1\right)$ if $\partial=0$ (resp. $\partial=1$ ).

Let $\Lambda$ be a character of $\mathcal{H}_{\nu}^{+}$and $(\lambda, \rho)$ the Satake parameter of $\Lambda$ $\left(\boldsymbol{\lambda}=\left(\lambda_{1}, \ldots, \lambda_{\nu}\right), \lambda_{0}=\lambda_{0}(\rho)\right)$. We define the local standard $L$-function of $\Lambda$ by

$$
L_{\mathfrak{p}}(\Lambda ; s)=L_{\mathfrak{p}}^{0}(\Lambda ; s) A_{\rho, \mathfrak{p}}(s) \quad(s \in \mathbf{C}),
$$

where

$$
L_{\mathfrak{p}}^{0}(\Lambda ; s)=\prod_{j=1}^{\nu}\left\{\left(1-\lambda_{j}(p) q^{-s}\right)\left(1-\lambda_{j}^{-1}(p) q^{-s}\right)\right\}^{-1}
$$

and

$$
\text { (1.18) } A_{\rho, \mathfrak{p}}(s)
$$

$$
= \begin{cases}1 & \left(n_{0}, \partial\right)=(0,0) \text { or }(1,0) \\ 1+\lambda_{0} q^{-(s-1 / 2)} & \left(n_{0}, \partial\right)=(1,1) \\ \left(1-q^{-2 s}\right)^{-1} & \left(n_{0}, \partial\right)=(2,0) \\ \left(1-\lambda_{0} q^{-s}\right)^{-1} & \left(n_{0}, \partial\right)=(2,1) \\ \left(1-\lambda_{0} q^{-s}\right)^{-1}\left(1+\lambda_{0} q^{-(s-1)}\right) & \left(n_{0}, \partial\right)=(2,2) \\ \left(1-\lambda_{0} q^{-(s+1 / 2)}\right)^{-1} & \left(n_{0}, \partial\right)=(3,1) \\ \left(1-\lambda_{0} q^{-(s+1 / 2)}\right)^{-1}\left(1+\lambda_{0} q^{-(s-1 / 2)}\right) & \left(n_{0}, \partial\right)=(3,2) \\ \left(1-\lambda_{0} q^{-s}\right)^{-1}\left(1-\lambda_{0} q^{-(s+1)}\right)^{-1} & \left(n_{0}, \partial\right)=(4,2) \text { and } \lambda_{0} \neq 0 \\ \left(1-q^{-2 s}\right)^{-1} & \left(n_{0}, \partial\right)=(4,2) \text { and } \lambda_{0}=0\end{cases}
$$

Note that our definition agrees with $[4,(1.6)-(1.8)]$ in the case $\lambda_{0}=1$.

The rest of this section is devoted to the calculation of a certain integral, which will be used to study the constant term of Eisenstein series in $§ 4.2$. Let $\boldsymbol{\mu}=\left(\mu_{1}, \ldots, \mu_{\nu+1}\right)$ be a $(\nu+1)$-tuple of unramified characters of $k^{\times}$and $\rho$ an irreducible representation of $E=K_{0} / K_{0}^{*}$. For an $x \in V_{\nu}=k^{2 \nu+n_{0}}$, we 
put

$$
\bar{n}(x)=\left[\begin{array}{ccc}
1 & & \\
x & 1_{2 \nu+n_{0}} & \\
-S_{\nu}[x] / 2 & -{ }^{t} x S_{\nu} & 1
\end{array}\right] \in G_{\nu+1}
$$

THEOREM 1.9. When $\left|\mu_{1}(p)\right|<\left|\mu_{j+1}(p)\right|<\left|\mu_{1}(p)^{-1}\right|(1 \leq j \leq \nu)$,

$$
\begin{gathered}
\int_{V_{\nu}} \phi_{\boldsymbol{\mu}, \rho}(\bar{n}(x)) d x=\phi_{\boldsymbol{\mu}, \rho}(1) \prod_{j=1}^{\nu} \frac{1-q^{-1}\left(\mu_{1} \mu_{j+1}^{-1}\right)(p)}{1-\left(\mu_{1} \mu_{j+1}^{-1}\right)(p)} \frac{1-q^{-1}\left(\mu_{1} \mu_{j+1}\right)(p)}{1-\left(\mu_{1} \mu_{j+1}\right)(p)} \\
\quad \times \frac{1}{1-\mu_{1}^{2}(p)} \begin{cases}\left(1+\mu_{0} \mu_{1}(p) q^{\partial-n_{0} / 2}\right)\left(1-\mu_{0} \mu_{1}(p) q^{-n_{0} / 2}\right) & \mu_{0} \neq 0 \\
1-\mu_{1}^{2}(p) q^{\partial-n_{0}} & \mu_{0}=0\end{cases}
\end{gathered}
$$

where $\phi_{\boldsymbol{\mu}, \rho}\left(\right.$ resp. $\left.\mu_{0}=\lambda_{0}(\rho)\right)$ is defined in (1.11) (resp.(1.15)) and the Haar measure $d x$ on $V_{\nu}$ is normalized so that the total volume of $L_{\nu}$ is 1 .

The following corollary is a direct consequence of Theorem 1.9 and the definition (1.16) of the standard $L$-function.

COROLlaRY 1.10. Let $\Lambda$ be a character of $\mathcal{H}_{\nu}^{+}$and $(\boldsymbol{\lambda}, \rho)$ its Satake parameter. Let $\boldsymbol{\mu}$ be $a(\nu+1)$-tuple of unramified characters defined by $\boldsymbol{\mu}=\left(|*|^{s}, \boldsymbol{\lambda}\right)(s \in \mathbf{C})$. Then

$$
\int_{V_{\nu}} \phi_{\boldsymbol{\mu}, \rho}(\bar{n}(x)) d x=\phi_{\boldsymbol{\mu}, \rho}(1) \frac{L_{\mathfrak{p}}(\Lambda ; s)}{L_{\mathfrak{p}}(\Lambda ; s+1)} \times\left\{\begin{array}{cl}
1 & n_{0}: \text { even } \\
\frac{\zeta_{\mathfrak{p}}(2 s)}{\zeta_{\mathfrak{p}}(2 s+1)} & n_{0}: \text { odd }
\end{array}\right.
$$

where $\zeta_{\mathfrak{p}}(s)=\left(1-q^{-s}\right)^{-1}$

Proof of Theorem 1.9. We shall demonstrate our assertion following the argument of Langlands $[3, \S 5]$. We introduce several subgroups of $\bar{N}=$ $\left\{\bar{n}(x) \mid x \in V_{\nu}\right\}$ :

$$
\begin{aligned}
& \overline{N_{i}}=\left\{\bar{n}\left(\left[\begin{array}{l}
y \\
x \\
0
\end{array}\right]\right) \mid y \in k^{i}, x \in V_{\nu-i}\right\} \quad \text { and } \\
& \overline{N_{i}^{\prime}}=\left\{\bar{n}\left(\left[\begin{array}{l}
y \\
0 \\
0
\end{array}\right]\right) \mid y \in k^{i}\right\} \quad(0 \leq i \leq \nu) .
\end{aligned}
$$

Then

$$
\bar{N}=\overline{N_{0}} \supset \overline{N_{1}} \supset \cdots \supset \overline{N_{\nu}} \supset \overline{N_{\nu}^{\prime}} \supset \overline{N_{\nu-1}^{\prime}} \supset \cdots \supset \overline{N_{1}^{\prime}} \supset \overline{N_{0}^{\prime}}=\{1\} .
$$


For these subgroups $\bar{M}$ of $\bar{N}$, we put

$$
\Delta_{\boldsymbol{\mu}, \rho}(\bar{M})=\int_{\bar{M}} \phi_{\boldsymbol{\mu}, \rho}(\bar{m}) d \bar{m}
$$

where the Haar measure $d \bar{m}$ is normalized so that the total volume of $\bar{M} \cap$ $K_{\nu+1}^{*}$ is 1 . First using the decomposition

$$
\begin{array}{r}
{\left[\begin{array}{cccc}
1 & & & \\
0 & 1 & & \\
v & 0 & 1 & \\
0 & -v & 0 & 1
\end{array}\right]=\left[\begin{array}{cccc}
v^{-1} & 0 & 1 & 0 \\
& v^{-1} & 0 & -1 \\
& & v & 0 \\
& & v
\end{array}\right]\left[\begin{array}{cccc} 
& & -1 & 0 \\
0 & 0 & v^{-1} & 0 \\
0 & -1 & 0 & v^{-1}
\end{array}\right]} \\
\\
(v \in k-\mathfrak{o})
\end{array}
$$

we have

$$
\Delta_{\boldsymbol{\mu}, \rho}\left(\overline{N_{j-1}}\right)=\Delta_{\boldsymbol{\mu}, \rho}\left(\overline{N_{j}}\right) \times \frac{1-q^{-1}\left(\mu_{1} \mu_{j+1}\right)(p)}{1-\left(\mu_{1} \mu_{j+1}\right)(p)} \quad(1 \leq j \leq \nu) .
$$

Secondly we consider the relation between $\Delta_{\mu, \rho}\left(\overline{N_{\nu}}\right)$ and $\Delta_{\mu, \rho}\left(\overline{N_{\nu}^{\prime}}\right)$. Note that for $x \in V_{0}-L_{0}$,

$$
\bar{n}\left(\left[\begin{array}{l}
0 \\
x \\
0
\end{array}\right]\right)=p(x) k(x)
$$

where

$$
\begin{gathered}
p(x)=\left[\begin{array}{ccccc}
z_{x}^{-1} & 0 & z_{x}^{-1 t} x S_{0} & 0 & -1 \\
& 1_{\nu} & 0 & 0 & 0 \\
& & 1_{n_{0}} & 0 & -x \\
& & & 1_{\nu} & 0 \\
& & & & z_{x}
\end{array}\right] \in N_{\nu} A_{\nu} \\
k(x)=\left[\begin{array}{ccccc}
0 & 0 & 0 & 0 & -1 \\
0 & 1_{\nu} & 0 & 0 & 0 \\
0 & 0 & \varepsilon_{x} & 0 & z_{x}^{-1} x \\
0 & 0 & 0 & 1_{\nu} & 0 \\
-1 & 0 & z_{x}^{-1 t} x S_{0} \varepsilon_{x} & 0 & z_{x}^{-1}
\end{array}\right] \in K_{\nu}, \\
z_{x}=S_{0}[x] / 2 \text { and } \varepsilon_{x}=1-z_{x}^{-1} x^{t} x S_{0} \in K_{0} . \text { Since } \\
p(x)^{-1} \bar{n}\left(\left[\begin{array}{l}
y \\
0 \\
0
\end{array}\right]\right) p(x)
\end{gathered}
$$




$$
=\left[\begin{array}{ccccc}
1 & 0 & 0 & { }^{t} y J_{\nu} & 0 \\
& 1_{\nu} & y z_{x}^{-1 t} x S_{0} & -y z_{x}^{-1 t} y J_{\nu} & -y \\
& & 1_{n_{0}} & -x z_{x}{ }^{t} y J_{\nu} & 0 \\
& & & 1_{\nu} & 0 \\
& & & & 1
\end{array}\right] \bar{n}\left(\left[\begin{array}{c}
y z_{x}^{-1} \\
0 \\
0
\end{array}\right]\right) \quad\left(y \in k^{\nu}\right)
$$

we have

$$
\begin{aligned}
\Delta_{\boldsymbol{\mu}, \rho}\left(\overline{N_{\nu}}\right) & =\Delta_{\boldsymbol{\mu}, \rho}\left(\overline{N_{\nu}^{\prime}}\right) \times\left\{1+\int_{V_{0}-L_{0}} \mu_{1}\left(z_{x}^{-1}\right)\left|z_{x}\right|^{-n_{0} / 2} \rho\left(\varepsilon_{x}\right) d x\right\} \\
& =\Delta_{\boldsymbol{\mu}, \rho}\left(\overline{N_{\nu}^{\prime}}\right) \times\left\{1+q^{-n_{0} / 2} \mu_{1}(p) \sum_{l=0}^{\infty} \mu_{1}\left(p^{2}\right)^{l} A_{0}+\sum_{l=1}^{\infty} \mu_{1}\left(p^{2}\right)^{l} A_{1}\right\}
\end{aligned}
$$

where we set

$$
A_{0} \quad \int_{L_{0}^{\prime}-L_{0}} \rho\left(1-z_{x}^{-1} x^{t} x S_{0}\right) d x \quad \text { and } \quad A_{1}=\int_{L_{0}-L_{0}^{\prime} p} \rho\left(1-z_{x}^{-1} x^{t} x S_{0}\right) d x .
$$

The next lemma will be proved at the end of this section.

LEMMA 1.11. Notation being as above, we have $A_{0}=\left(q^{\partial}-1\right) \mu_{0}$ and $A_{1}=1-q^{\partial-n_{0}}$.

Therefore we get

$$
\begin{aligned}
& \text { 23) } \Delta_{\boldsymbol{\mu}, \rho}\left(\overline{N_{\nu}}\right) \\
& =\frac{\Delta_{\boldsymbol{\mu}, \rho}\left(\overline{N_{\nu}^{\prime}}\right)}{1-\mu_{1}(p)^{2}} \begin{cases}\left(1+\mu_{0} \mu_{1}(p) q^{\partial-n_{0} / 2}\right)\left(1-\mu_{0} \mu_{1}(p) q^{-n_{0} / 2}\right) & \mu_{0} \neq 0 \\
\left(1-\mu_{1}(p)^{2} q^{\partial-n_{0}}\right) & \mu_{0}=0\end{cases}
\end{aligned}
$$

Finally taking account of

$$
\left[\begin{array}{ll}
1 & 0 \\
v & 1
\end{array}\right]=\left[\begin{array}{cc}
v^{-1} & 1 \\
0 & v
\end{array}\right]\left[\begin{array}{cc}
0 & -1 \\
1 & v^{-1}
\end{array}\right] \quad(v \in k-\mathfrak{o})
$$

we have

$$
\Delta_{\boldsymbol{\mu}, \rho}\left(\overline{N_{j}^{\prime}}\right)=\Delta_{\boldsymbol{\mu}, \rho}\left(\overline{N_{j-1}^{\prime}}\right) \frac{1-q^{-1}\left(\mu_{1} \mu_{j+1}^{-1}\right)(p)}{1-\left(\mu_{1} \mu_{j+1}^{-1}\right)(p)} \quad(1 \leq j \leq \nu) .
$$

Combining $(1.22)-(1.24)$, we get the theorem. 
Poof of Lemma 1.11. Take any $x \in L_{0}-L_{0}^{\prime} p$ and $\alpha \in L_{0}^{*}$. Since $z_{x} \in \mathfrak{o}^{\times}$, we have $\left(1-\varepsilon_{x}\right) \alpha=z_{x}^{-1} x^{t} x S \alpha \in L_{0}$, and hence we have $\varepsilon_{x} \in K_{0}^{*}$ and $A_{1}=\operatorname{vol}\left(L_{0}-L_{0}^{\prime} p\right)=1-q^{\partial-n_{0}}$. Now we consider $A_{0}$. It is easily seen that $\varepsilon_{x} K_{0}^{*}=\varepsilon_{x+y} K_{0}^{*}$ for $x \in L_{0}^{\prime}-L_{0}$ and $y \in L_{0}$. Thus we have

$$
A_{0}=\sum_{x \in\left(L_{0}^{\prime}-L_{0}\right) / L_{0}} \rho\left(\varepsilon_{x}\right)
$$

We evaluate the sum by a case-by-case argument. For example, we consider the case $\left(n_{0}, \partial\right)=(2,2)$ and use the same notation in the proof of Proposition 1.1. For $x=\left[\begin{array}{l}x_{1} \\ x_{2}\end{array}\right] p^{-1}$ in $L_{0}^{\prime}-L_{0}$, we have

$$
\varepsilon_{x}=\left[\begin{array}{cc}
u & b u+c v \\
v & -u
\end{array}\right], \quad u=\frac{-x_{1}^{2}+c x_{2}^{2}}{x_{1}^{2}+b x_{1} x_{2}+c x_{2}^{2}}, \quad v=\frac{-2 x_{1} x_{2}-b x_{2}^{2}}{x_{1}^{2}+b x_{1} x_{2}+c x_{2}^{2}} .
$$

Being $C$ the unique cyclic normal subgroup of $E=D_{q+1}$ of index 2 , we know

$$
A_{0}=(q-1) \sum_{g \in E-C} \rho(g) .
$$

Therefore our assertion follows from the definition of $\mu_{0}$ in (1.15).

\section{$\S 2$. Norm functions}

\subsection{Definition and properties of norm functions}

Keep notation in the previous section. First we introduce a function $\mu$ on $M_{m}(k)$ by

$$
\mu(g)=\sum_{e_{i}<0}\left|e_{i}\right|
$$

where $\left(p^{e_{1}}, \ldots, p^{e_{r}}, 0, \ldots, 0\right)$ is the elementary divisor of $g$ in $M_{m}(k)$. Note that $\mathfrak{p}^{-\mu(g)}$ coincides with the $\mathfrak{o}$-module generated by $\mathfrak{o}$ and all minors of $g$. The following properties are elementary.

LEMMA 2.1. (i) $\mu(g) \leq \mu$ if and only if there exists an element $A \in M_{m}(\mathfrak{o})$ such that $\operatorname{ord} \mathfrak{p} \operatorname{det} A=\mu$ and $A g$ is in $M_{m}(\mathfrak{o})$.

(ii) For $g_{1}, g_{2} \in M_{m}(k), \mu\left(g_{1} g_{2}\right) \leq \mu\left(g_{1}\right)+\mu\left(g_{2}\right)$.

(iii) For $g \in M_{m}(k)$ and $g^{\prime} \in M_{m^{\prime}}(k), \mu\left(\left[\begin{array}{ll}g & * \\ 0 & g^{\prime}\end{array}\right]\right) \geq \mu(g)+\mu\left(g^{\prime}\right)$.

(iv) For $g \in M_{m}(k)$ and $A \in M_{m}(\mathfrak{o}), \mu(g+A)=\mu(g)$. 
Let $S$ be a non-degenerate even integral symmetric matrix of rank $m$ and $G$ the orthogonal group of $S$. We set $K=G \cap G L_{m}(\mathfrak{o})$ and $K^{*}=\{u \in$ $\left.K \mid(u-1) S^{-1} \in M_{m}(\mathfrak{o})\right\}$. We do not suppose the maximality of $S$ in this subsection. We define the norm function $\mu_{G}$ on $G$ by

$$
\mu_{G}(g)=\mu\left((g-1) S^{-1}\right) \quad \text { for } g \in G \text {. }
$$

Evidently $\mu_{G}(g)=0$ if and only if $g \in K^{*}$.

Proposition 2.2. (i) $\mu_{G}\left(g^{-1}\right)=\mu_{G}(g)$ for $g \in G$.

(ii) $\mu_{G}$ is bi-K*-invariant and $\mu_{G}\left(u^{-1} g u\right)=\mu_{G}(g)$ for $u \in K$.

(iii) For $g_{1}, g_{2} \in G, \mu_{G}\left(g_{1} g_{2}\right) \leq \mu_{G}\left(g_{1}\right)+\mu_{G}\left(g_{2}\right)$.

(iv) $\quad$ We put $S_{1}=\left[\begin{array}{ccc} & S & 1 \\ 1 & \end{array}\right]$ and $G_{1}=O\left(S_{1}\right)$. Then for $\alpha \in k^{\times}$and $\beta \in G$,

$$
\mu_{G_{1}}\left(\left[\begin{array}{ccc}
\alpha & * & * \\
& \beta & \alpha^{-1}
\end{array}\right]\right) \geq \mu_{G}(\beta)+\left|\operatorname{ord}_{\mathfrak{p}} \alpha\right| \text {. }
$$

Proof. Since $\left(g^{-1}-1\right) S^{-1}={ }^{t}\left((g-1) S^{-1}\right)$, we get (i) from the definition of $\mu$. For any $u_{1}, u_{2} \in K^{*},\left(u_{1} g u_{2}-1\right) S^{-1}=u_{1}(g-1) u_{2} S^{-1}+\left(u_{1} u_{2}-1\right) S^{-1}$. Thus from Lemma 2.1 (iv), we have $\mu_{G}\left(u_{1} g u_{2}\right)=\mu\left(u_{1}(g-1) S^{-1 t} u_{2}^{-1}\right)=$ $\mu_{G}(g)$. Similarly for $u \in K, \mu_{G}\left(u^{-1} g u\right)=\mu\left(u^{-1}(g-1) u S^{-1}\right)=\mu((g-$ 1) $\left.S^{-1 t} u^{-1}\right)=\mu_{G}(g)$ and we obtain (ii). To see (iii) we take an $A_{1}$ (resp. $\left.A_{2}\right)$ in $M_{m}(\mathfrak{o})$ such that $\operatorname{ord}_{\mathfrak{p}} \operatorname{det} A_{1}=\mu_{G}\left(g_{1}\right)\left(\operatorname{resp} . \operatorname{ord}_{\mathfrak{p}} \operatorname{det} A_{2}=\mu_{G}\left(g_{2}\right)\right)$ and $A_{1}\left(g_{1}-1\right) S^{-1} \in M_{m}(\mathfrak{o})$ (resp. $\left.\left(g_{2}-1\right) S^{-1} A_{2} \in M_{m}(\mathfrak{o})\right)$. Then

$$
\begin{aligned}
B & =A_{1}\left(g_{1} g_{2}-1\right) S^{-1} A_{2} \\
& =A_{1}\left(g_{1}-1\right) S^{-1} S\left(g_{2}-1\right) S^{-1} A_{2}+A_{1}\left(g_{1}-1\right) S^{-1} A_{2}+A_{1}\left(g_{2}-1\right) S^{-1} A_{2}
\end{aligned}
$$

is in $M_{m}(\mathfrak{o})$ and hence $\mu_{G}\left(g_{1} g_{2}\right)=\mu\left(A_{1}^{-1} B A_{2}^{-1}\right) \leq \mu\left(A_{1}^{-1}\right)+\mu(B)+$ $\mu\left(A_{2}^{-1}\right)=\mu_{G}\left(g_{1}\right)+\mu_{G}\left(g_{2}\right)$. Finally by using Lemma 2.1 (iii), we get

$$
\begin{aligned}
& \mu_{G_{1}}\left(\left[\begin{array}{ccc}
\alpha & * & * \\
& \beta & { }^{*} \\
& & \alpha^{-1}
\end{array}\right]\right)=\mu\left(\left[\begin{array}{ccc}
\alpha-1 & { }^{*} & * \\
& (\beta-1) S^{-1} & \alpha^{-1^{*}}-1
\end{array}\right]\right) \\
& \geq \mu_{G}(\beta)+\mu(\alpha-1)+\mu\left(\alpha^{-1}-1\right)=\mu_{G}(\beta)+\left|\operatorname{ord}_{\mathfrak{p}} \alpha\right|
\end{aligned}
$$

and our assertions are proved completely. 
Now we take a non-degenerate even integral symmetric matrix $T=$ $\left[\begin{array}{cc}S & -S \alpha \\ -{ }^{t} \alpha S & -2 a\end{array}\right]$ of rank $m+1$ and put $H=O(T)$. We embed $G$ into $H$ through $\iota_{0}$ given by

$$
\iota_{0}(g)=\left[\begin{array}{cc}
g & (1-g) \alpha \\
0 & 1
\end{array}\right] \quad(g \in G) .
$$

Proposition 2.3. For $g \in G, \mu_{G}(g)=\mu_{H}\left(\iota_{0}(g)\right)$. In particular, $\iota_{0}\left(K^{*}\right)=U^{*} \cap \iota_{0}(G)$, where $U^{*}=\left\{u \in H \mid(u-1) T^{-1} \in M_{m+1}(\mathfrak{o})\right\}$.

Proof. Since $T^{-1}=\left[\begin{array}{cc}1 & \alpha \\ 0 & 1\end{array}\right]\left[\begin{array}{cc}S^{-1} & 0 \\ 0 & -(2 a+S[\alpha])^{-1}\end{array}\right]\left[\begin{array}{cc}1 & 0 \\ t^{t} \alpha & 1\end{array}\right]$, we get $\left(\iota_{0}(g)-1\right) T^{-1}=\left[\begin{array}{cc}(g-1) S^{-1} & 0 \\ 0 & 0\end{array}\right]$ and hence our assertion is verified.

Remark 2.4. In [4, Lemma 4.3], we proved $\mu(g)=\mu\left(\iota_{0}(g)\right)$ under a certain condition, which is not satisfied in general. This is a reason why we now use $\mu_{G}$ instead of $\mu$.

\subsection{Parametrization}

From now on we assume that $S$ is maximal in the sense of $\S 1.1$. Put $L=\mathfrak{o}^{m} \subset V=k^{m}$ and denote by $L^{*}$ the dual lattice of $L$. For a primitive element $\eta \in L^{*}$ with $S[\eta] \neq 0$, we put

$$
V_{\eta}=\{x \in V \mid S(\eta, x)=0\} \supset L_{\eta}=L \cap V_{\eta} .
$$

We denote by $G_{\eta}$ the stabilizer subgroup of $\eta$ in $G$. Our aim in this subsection is to give a double coset decomposition $G_{\eta} \backslash G / K^{*}$ explicitly. For $l \in \mathbf{Z}$ we put

$$
G(\eta ; l)=\left\{g \in G \mid g^{-1} \eta \cdot p^{l} \in L_{\mathrm{prm}}^{*}\right\},
$$

where $L_{\mathrm{prm}}^{*}$ stands for the set consisting of all primitive elements in $L^{*}$. Clearly $G(\eta ; l)$ is left- $G_{\eta}$, right- $K$ invariant.

LEMMA 2.5. Assume that $L_{\eta}$ is a maximal o-integral lattice with respect to the restriction of $S$. Then
(i) $\quad G=\coprod_{l \geq 0} G(\eta ; l)$.
(ii) $\operatorname{Min}_{g \in G(\eta ; l)} \mu_{G}(g)=l$. 
Proof. The first part was shown in [4, Proposition 3.9]. We shall prove (ii) for $l \geq 1$ (the assertion is trivial for $l=0$ ). For $g \in G(\eta ; l)$, $\left(g^{-1}-1\right) S^{-1} \cdot S \eta=g^{-1} \eta-\eta \notin L \cdot p^{-(l-1)}$ and hence $\left(g^{-1}-1\right) S^{-1} \notin$ $M_{m}(\mathfrak{o}) \cdot p^{-(l-1)}$. Thus we know that $\mu_{G}(g)=\mu\left(\left(g^{-1}-1\right) S^{-1}\right) \geq l$. Let us show the existence of $g \in G(\eta ; l)$ such that $\mu_{G}(g)=l$. Taking a suitable o-basis of $L$ we may assume $S=\left[\begin{array}{lll} & S^{\prime} & 1 \\ 1 & & \end{array}\right]$ and $\eta=\left[\begin{array}{l}a \\ \alpha \\ 1\end{array}\right]$, because $G \neq K$ implies that $S$ is isotropic. Then $M_{l}=\left[\begin{array}{ccc}p^{-l} & & \\ & 1 & \\ & & p^{l}\end{array}\right]$ has the required property.

We set

$$
\begin{aligned}
& K^{(\eta)}=\{u \in K \mid(u-1) \eta \in L\} \quad \text { and } \\
& L^{(\eta)}=\left\{\eta^{\prime} \in L^{*} \mid \frac{1}{2} S\left[\eta^{\prime}\right] \equiv \frac{1}{2} S[\eta] \quad(\bmod \mathfrak{o})\right\}
\end{aligned}
$$

It is clear that $K^{(\eta)}$ is a subgroup of $K$ containing $K^{*}$ and $L^{(\eta)}$ is a union of several $L$-cosets in $L^{*}$. For $x \in V$ with $S[x] \neq 0$, we put

$$
z_{x}=\frac{1}{2} S[x] \in k^{\times} \quad \text { and } \quad \varepsilon_{x}=1-z_{x}^{-1} x^{t} x S \in G .
$$

LEMMA 2.6. The mapping $\varphi: u \mapsto u \eta$ gives a bijection between $K / K^{(\eta)}$ and $L^{(\eta)} / L$.

Proof. Clearly $\varphi$ is well-defined. If $\varphi(u)=\varphi\left(u^{\prime}\right)$ for $u, u^{\prime} \in K$, then $u^{-1} u^{\prime} \eta-\eta=u^{-1}\left(u^{\prime} \eta-u \eta\right) \in L$. So we know that $\varphi$ is injective. Take any $\eta^{\prime} \in L^{(\eta)}$ such that $x=\eta-\eta^{\prime} \notin L$. Then we easily know that $x \in L p^{-1}$, $z_{x} \in p^{-1} \mathfrak{o}^{\times}$and $\varepsilon_{x} \in K$. Since

$$
\varphi\left(\varepsilon_{x}\right)=\eta-z_{x}^{-1} x\left(z_{x}-z_{\eta^{\prime}}+z_{\eta}\right) \equiv \eta^{\prime} \quad(\bmod L),
$$

we get the surjectivity of $\varphi$.

Combining Proposition 3.9 in [4] with this, we obtain

Proposition 2.7. Let $S$ be maximal and $\eta$ primitive in $L^{*}$ with $S[\eta] \neq$ 0 . We assume that $L_{\eta}$ is a maximal $\mathfrak{0}$-integral lattice with respect to $S \mid L_{\eta}$. Then 
(i) $\quad G(\eta ; l)=G_{\eta} M_{l} K^{*} \quad(l \geq 1) \quad$ and

(ii) $\quad G(\eta ; 0)=\coprod_{u \in K^{(\eta)} \backslash K} G_{\eta} u K^{*}=\coprod_{\eta^{\prime} \in L^{(\eta)} / L, \eta-\eta^{\prime} \notin L} G_{\eta} \varepsilon_{\eta-\eta^{\prime}}^{-1} K^{*} \coprod G_{\eta} K^{*}$,

where $M_{l}$ is an element in $G(\eta ; l)$ such that $\mu_{G}\left(M_{l}\right)=l$ and $\varepsilon_{\eta-\eta^{\prime}}$ is defined in (2.7).

We say that $g \in G$ is minimal (with respect to $\eta$ ) if $\mu_{G}(g) \leq \mu_{G}\left(g_{0} g\right.$ ) for any $g_{0} \in G_{\eta}$. For $g \in G(\eta ; l)$ with $l \geq 1, g$ is minimal if and only if $\mu_{G}(g)=l$.

\subsection{Norm functions and the Iwasawa decomposition}

Let $S$ (resp. $T=\left[\begin{array}{cc}S & -S \alpha \\ -{ }^{t} \alpha S & -2 a\end{array}\right]$ ) be a maximal even integral symmetric matrix of rank $m$ (resp. $m+1$ ). Then $S_{1}=\left[\begin{array}{lll} & S^{1}\end{array}\right]$ is also maximal. We put

$$
\begin{aligned}
& L=\mathfrak{o}^{m}, \quad M=\left[\begin{array}{l}
L \\
\mathfrak{o}
\end{array}\right]=\mathfrak{o}^{m+1}, \quad L_{1}=\left[\begin{array}{c}
\mathfrak{o} \\
L \\
\mathfrak{o}
\end{array}\right]=\mathfrak{o}^{m+2}, \\
& V=L \otimes_{\mathfrak{o}} k, \quad W=M \otimes_{\mathfrak{o}} k, \quad V_{1}=L_{1} \otimes_{\mathfrak{o}} k, \\
& G=O(S), \quad K=G \cap G L_{m}(\mathfrak{o}), \\
& K^{*}=\left\{u \in K \mid(u-1) S^{-1} \in M_{m}(\mathfrak{o})\right\} \text {, } \\
& H=O(T), \quad U=H \cap G L_{m+1}(\mathfrak{o}), \\
& U^{*}=\left\{u \in U \mid(u-1) T^{-1} \in M_{m+1}(\mathfrak{o})\right\}, \\
& G_{1}=O\left(S_{1}\right), K_{1}=G_{1} \cap G L_{m+2}(\mathfrak{o}) \text {, } \\
& K_{1}^{*}=\left\{u \in K_{1} \mid(u-1) S_{1}^{-1} \in M_{m+2}(\mathfrak{o})\right\}, \\
& \eta=\left[\begin{array}{c}
a \\
\alpha \\
1
\end{array}\right] \in L_{1}^{*}=S_{1}^{-1} L_{1}, \quad \Delta=2 a+S[\alpha] \in k^{\times}, \\
& \xi=\left[\begin{array}{c}
\alpha \\
1
\end{array}\right] \Delta^{-1} \in M^{*}=T^{-1} M
\end{aligned}
$$

We embed $V$ into $W$ (resp. $W$ into $V_{1}$ ) by $j_{0}$ (resp. $j$ ) :

$$
j_{0}(y)=\left[\begin{array}{l}
y \\
0
\end{array}\right] \quad \text { and } \quad j\left(\left[\begin{array}{l}
y \\
z
\end{array}\right]\right)=\left[\begin{array}{c}
-a z-\underset{S}{S}(\alpha, y) \\
z
\end{array}\right] \quad(y \in V, z \in k)
$$


Then $j_{0}(V)$ (resp. $j(W)$ ) is the orthogonal complement of $\xi$ (resp. $\eta$ ) in $W$ (resp. $V_{1}$ ). We define embeddings $\iota_{0}: G \longrightarrow H$ and $\iota: H \longrightarrow G_{1}$ to be

$$
\begin{aligned}
\iota_{0}(g)\left(\xi t+j_{0}(y)\right) & =\xi t+j_{0}(g y) & & (g \in G, y \in V, t \in k), \\
\iota(h)(\eta t+j(w)) & =\eta t+j(h w) & & (h \in H, w \in W, t \in k) .
\end{aligned}
$$

Then $\iota_{0}(G)$ (resp. $\left.\iota(H)\right)$ coincides with the stabilizer subgroup $H_{\xi}$ of $\xi$ in $H$ (resp. $G_{1, \eta}$ of $\eta$ in $\left.G_{1}\right)$. Since

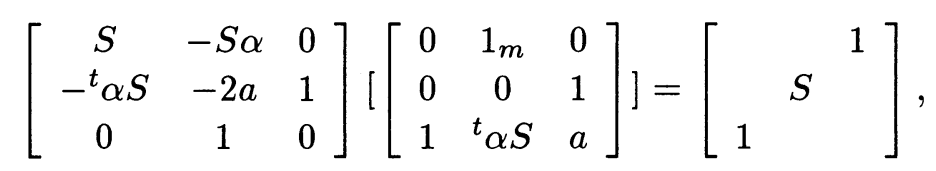

Proposition 2.3 implies that

$$
\iota_{0}\left(K^{*}\right)=U^{*} \cap \iota_{0}(G) \text { and } \iota\left(U^{*}\right)=K_{1}^{*} \cap \iota(H) .
$$

From the Iwasawa decomposition, any $g \in G_{1}$ can be written in the form

$$
\begin{aligned}
& g_{1}=\left[\begin{array}{rrr}
\alpha\left(g_{1}\right) & \stackrel{*}{*} & * \\
& \beta\left(g_{1}\right) & \stackrel{*}{*} \\
& \alpha\left(g_{1}\right)^{-1}
\end{array}\right] u\left(g_{1}\right) \\
&\left(\alpha\left(g_{1}\right) \in k^{\times}, \beta\left(g_{1}\right) \in G, u\left(g_{1}\right) \in K_{1}^{*}\right)
\end{aligned}
$$

The following theorem is a refinement of Lemma $\mathrm{A}$ in [4].

THEOREM 2.8. (i) Let $M \in H$ be minimal with respect to $\xi$. Then for any $g \in G, \mu_{H}\left(\iota_{0}(g) M\right)=\mu_{G}(g)+\mu_{H}(M)$.

(ii) For any $h \in H$, we have $\mu_{H}(h)=\mu_{G}(\beta(\iota(h)))+\operatorname{ord}_{\mathfrak{p}} \alpha(\iota(h))$.

Proof. We put $\alpha_{M}=\alpha(\iota(M)) \in k^{\times}$and $\beta_{M}=\beta(\iota(M)) \in G$. Because of $\iota(M)^{-1} \eta=\eta$, we have $\alpha_{M} \in \mathfrak{o}$. By Proposition 2.2 (iv) and Proposition 2.3 , we have

$$
\operatorname{ord}_{p}\left(\alpha_{M}\right)+\mu_{G}\left(\beta_{M}\right) \leq \mu_{H}(M)
$$

Noting that

$(2.14) \iota\left(\iota_{0}(g)\right)=\left[\begin{array}{ccc}1 & -{ }^{t} \alpha\left({ }^{t} g^{-1}-1\right) S & S\left(\left(g^{-1}-1\right) \alpha, \alpha\right) \\ & g & (1-g) \alpha \\ & 1\end{array}\right]$ for $g \in G$, 
we can write

$$
\begin{array}{r}
\iota\left(\iota_{0}\left(\beta_{M}\right)^{-1} M\right)=\left[\begin{array}{ccc}
\alpha_{M} & & \\
& 1 & \\
& & \alpha_{M}^{-1}
\end{array}\right]\left[\begin{array}{ccc}
1 & -{ }^{t} x_{M} S & -S\left[x_{M}\right] / 2 \\
& 1 & x_{M} \\
& 1
\end{array}\right] u_{M} \\
\\
\left(x_{M} \in V, u_{M} \in K_{1}^{*}\right) .
\end{array}
$$

Since $u_{M} \eta-\eta \in L_{1}, x_{M} \alpha_{M}$ must be in $L$ and hence

$$
\iota\left(\iota_{0}\left(\beta_{M}^{-1}\right) M\right) \in K_{1}^{*}\left[\begin{array}{lll}
\alpha_{M} & & \\
& 1 & \\
& & \alpha_{M}^{-1}
\end{array}\right] K_{1}^{*} .
$$

From Proposition 2.2, Proposition 2.3 and the minimality of $M$, we have

$$
\operatorname{ord}_{\mathfrak{p}}\left(\alpha_{M}\right)=\mu_{H}\left(\iota_{0}\left(\beta_{M}\right)^{-1} M\right) \geq \mu_{H}(M) \text {. }
$$

Inequalities (2.13) and (2.15) implies

$$
\operatorname{ord}_{\mathfrak{p}}\left(\alpha_{M}\right)=\mu_{H}(M) \text { and } \mu_{G}\left(\beta_{M}\right)=0 \text { (i.e. } \beta_{M} \in K^{*} \text { ). }
$$

Therefore for $g \in G$,

$$
\iota\left(\iota_{0}(g) M\right) \in\left[\begin{array}{ccc}
\alpha_{M} & * & * \\
& g & \alpha_{M}^{*}
\end{array}\right] K_{1}^{*}
$$

and hence $\mu_{H}(M)+\mu_{G}(g) \leq \mu_{H}\left(\iota_{0}(g) M\right)$. On the other hand, Proposition 2.2 (iii) implies the inequality $\mu_{H}(M)+\mu_{G}(g) \geq \mu_{H}\left(\iota_{0}(g) M\right)$. Thus we have proved the first part. Because of Proposition 2.7, to see (ii) we may assume $h=\iota_{0}(g) M$. Then from the above discussion, $\beta(\iota(h)) \in g K^{*}$ and $\alpha(\iota(h)) \in \alpha_{M} \mathfrak{o}^{\times}$and hence the identity (ii) holds.

\subsection{Construction of the standard $L$-function by the norm function}

For $g \in G$ and $s \in \mathbf{C}$, we put

$$
N_{G, s}(g)=q^{-\mu_{G}(g) s} \text {. }
$$

Our purpose of this subsection is to give a formula corresponding to Lemma $\mathrm{B}$ in [4]. We start with a key proposition. 
Proposition 2.9. For $t \in k^{\times}$and $g \in G$,

$$
\begin{aligned}
f_{G_{1}}(t, g ; s) & \stackrel{\text { def }}{=} \int_{V} N_{G_{1}, s+m / 2}\left(\left[\begin{array}{lll}
t & & \\
& g & \\
& & t^{-1}
\end{array}\right] n_{G}(x)\right) d x \\
& =|t|^{-m / 2} q^{-\left|\operatorname{ord}_{\mathfrak{p}} t\right| s} N_{G, s-1+m / 2}(g) \frac{1-q^{-(2 s+m)}}{1-q^{-2 s}},
\end{aligned}
$$

where we put $n_{G}(x)=\left[\begin{array}{ccc}1 & -{ }^{t} x S & -S[x] / 2 \\ & 1 & x \\ & 1\end{array}\right] \in G_{1}$ for $x \in V=k^{m}$.

\section{Proof. Since}

$$
\left\{\begin{array}{l}
f_{G_{1}}\left(t \varepsilon, u_{1} g u_{2} ; s\right)=f_{G_{1}}(t, g ; s) \text { for }{ }^{\forall} \varepsilon \in \mathfrak{o}^{\times},{ }^{\forall} u_{1},{ }^{\forall} u_{2} \in K^{*} \\
f_{G_{1}}(t, g ; s)=|t|^{-m} f_{G_{1}}\left(t^{-1}, g^{-1} ; s\right),
\end{array}\right.
$$

it is sufficient to see that

(2.19) $f_{G_{1}}\left(p^{-\beta}, g ; s\right)$

$$
=q^{-\beta(s+m / 2)} N_{G, s-1+m / 2}(g) \frac{1-q^{-(2 s+m)}}{1-q^{-2 s}} \text { for } \beta \geq 0 .
$$

We first consider the case where $S$ is anisotropic. If $x \in V-L$, then

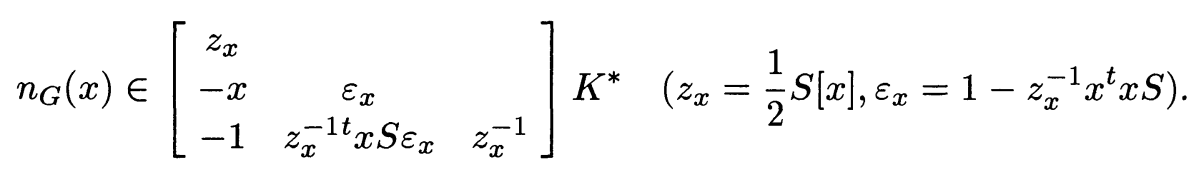

Thus for $g \in G=K$ and $\beta \geq 0$, we have

$$
\begin{aligned}
f_{G_{1}}\left(p^{-\beta}, g ; s\right)= & N_{G_{1}, s+m / 2}\left(\left[\begin{array}{lll}
p^{-\beta} & & \\
& g & p^{\beta}
\end{array}\right]\right) \\
& +\int_{V-L} N_{G_{1}, s+m / 2}\left(\left[\begin{array}{lll}
p^{-\beta} z_{x} & & \\
& g \varepsilon_{x} & p^{\beta} z_{x}^{-1}
\end{array}\right]\right) d x \\
= & q^{-\beta(s+m / 2)} N_{G, s+m / 2}(g) \\
& +\sum_{l=1}^{\infty}\left(1-q^{-m+\partial}\right) q^{l m-(\beta+2 l)(s+m / 2)} N_{G, s+m / 2}(g) \\
& +\sum_{l=0}^{\infty} q^{l m-(\beta+2 l+1)(s+m / 2)} \int_{L^{\prime}-L} N_{G, s+m / 2}\left(g \varepsilon_{x}\right) d x .
\end{aligned}
$$


A case-by-case argument implies that

$$
\begin{aligned}
& \int_{L^{\prime}-L} N_{G, s+m / 2}\left(g \varepsilon_{x}\right) d x \\
& = \begin{cases}0 & \text { if } \partial=0 \\
(q-1) q^{-(s+m / 2)} & \text { if } \partial=1 \text { and } g \in K^{*} \\
q-1 & \text { if } \partial=1 \text { and } g \notin K^{*} \\
\left(q^{2}-1\right) q^{-(s+m / 2)} & \text { if } \partial=2 \text { and } g \in C \\
(q-1)\left(q^{-2(s+m / 2)+1}+1\right) & \text { if } \partial=2 \text { and } g \notin C\end{cases}
\end{aligned}
$$

where $C$ is the unique normal cyclic subgroup of $K / K^{*}$ of index 2. Thus our assertion is true in this case. Now we shall prove $(2.19)$ by an induction on $m$. We assume that (2.19) holds for $m-1$ and $S$ is isotropic. We set $S=\left[\begin{array}{lll} & S^{\prime} & 1 \\ 1 & & \end{array}\right]$. We suppose that $g$ is not in $K$. Then there exists an $\eta=\left[\begin{array}{c}a \\ \alpha \\ 1\end{array}\right] \in L_{\mathrm{prm}}^{*}$ such that $g^{-1} \eta \notin L^{*}$ and $T=S \mid L_{\eta}$ is maximal. As is seen in the proof of Lemma $2.5, g \in G_{\eta} M_{\lambda} K^{*}$ with $M_{\lambda}=\left[\begin{array}{lll}p^{-\lambda} & & \\ & 1 & \\ & & p^{\lambda}\end{array}\right]$ and $\lambda=\mu_{G}(g)$. Note that $x_{0}=\left[\begin{array}{l}1 \\ 0 \\ 0\end{array}\right] \in L$ is isotropic and satisfies $S\left(\eta, x_{0}\right)=1$. We embed $H=O(T)$ into $G$ as the stabilizer subgroup of $\eta$ (cf. $\S 2.3$ ). Similarly the orthogonal group $H_{1}$ of $T_{1}=\left[\begin{array}{lll} & T^{1} \\ 1 & \end{array}\right]$ is embedded into $G_{1}$ as the stabilizer subgroup of $\eta^{\sim}=\left[\begin{array}{l}0 \\ \eta \\ 0\end{array}\right]$. For $h \in H=G_{\eta}$,

$$
\begin{aligned}
& f_{G_{1}}\left(p^{-\beta}, h M_{\lambda} ; s\right) \\
& =\int_{V_{\eta}} \int_{k} N_{G_{1}, s+m / 2}\left(\left[\begin{array}{lll}
p^{-\beta} & & \\
& h & \\
& & p^{\beta}
\end{array}\right] n_{G}\left(y+x_{0} u\right)\left[\begin{array}{lll}
1 & & \\
& M_{\lambda} & \\
& & 1
\end{array}\right]\right) d y d u \\
& =\int_{V_{\eta}} \int_{\mathfrak{p}^{-\lambda}} N_{G_{1}, s+m / 2}\left(\left[\begin{array}{llll}
p^{-\beta} & & \\
& h & \\
& & p^{\beta}
\end{array}\right] n_{G}(y)\left[\begin{array}{lll}
1 & & \\
& M_{\lambda} & \\
& & 1
\end{array}\right] n_{G}\left(M_{\lambda}^{-1} x_{0} u\right)\right) d y d u \\
& \quad+\sum_{l=\lambda+1}^{\infty} \int_{V_{\eta}} \int_{p^{-l} \mathfrak{o}^{\times}} N_{G_{1}, s+m / 2}\left(\left[\begin{array}{lll}
p^{-\beta} & & \\
& h & \\
& & p^{\beta}
\end{array}\right] n_{G}(y)\right.
\end{aligned}
$$




$$
\left.\times\left[\begin{array}{ccc}
p^{\lambda} u & & \\
& 1 & \\
& & p^{-\lambda} u^{-1}
\end{array}\right] g_{u}\right) d y d u
$$

where $g_{u}=\left[\begin{array}{lll}p^{-\lambda} u^{-1} & & \\ & M_{\lambda} & \\ & & p^{\lambda} u\end{array}\right] n_{G}\left(M_{\lambda}^{-1} x_{0} u\right)$. Since $g_{u}^{-1} \eta^{\sim}=\left[\begin{array}{c}u \\ M_{\lambda}^{-1} \eta \\ 0\end{array}\right]$
$\in L_{1, \mathrm{prm}}^{*} p^{-l}$ and

$$
\begin{aligned}
\mu_{G_{1}}\left(g_{u}\right) & =\mu\left(\left[\begin{array}{ccc}
p^{-\lambda} u^{-1}-1 & -{ }^{t}\left(M_{\lambda}^{-1} x_{0} p^{-\lambda}\right) & -S\left[x_{0}\right] p^{-\lambda} u / 2 \\
& \left(M_{\lambda}-1\right) S^{-1} & x_{0} u \\
& & p^{\lambda} u-1
\end{array}\right]\right) \\
& =\mu_{G}\left(M_{\lambda}\right)+l-\lambda,
\end{aligned}
$$

we know that $g_{u}$ is minimal with respect to $\eta^{\sim}$ and $\mu_{G_{1}}\left(g_{u}\right)=l$. Therefore by Theorem $2.8(\mathrm{i}), f_{G_{1}}\left(p^{-\beta}, h M_{\lambda} ; s\right)$ is equal to

$$
\begin{aligned}
& \int_{k^{m-1}} q^{\lambda} N_{H_{1}, s+m / 2}\left(\left[\begin{array}{lll}
p^{-\beta} & & \\
& h & \\
& & p^{\beta}
\end{array}\right] n_{H}(y)\right) q^{-\lambda(s+m / 2)} d y \\
& +\sum_{l=\lambda+1}^{\infty} q^{l}\left(1-q^{-1}\right) \int_{k^{m-1}} N_{H_{1}, s+m / 2}\left(\left[\begin{array}{lll}
p^{-(\beta+l-\lambda)} & & \\
& h & p^{\beta+l-\lambda}
\end{array}\right] n_{H}(y)\right) \\
& =q^{-\lambda(s-1+m / 2)}\left\{f_{H_{1}}\left(p^{-\beta}, h ; s+1 / 2\right)\right. \\
& \times q^{(l-\lambda)(m-1)-l(s+m / 2)} d y \\
& \left.+\sum_{l=\lambda+1}^{\infty}\left(1-q^{-1}\right) q^{(l-\lambda)(-s+m / 2)} f_{H_{1}}\left(p^{-(\beta+l-\lambda)}, h ; s+1 / 2\right)\right\} \text {. }
\end{aligned}
$$

Thus the induction assumption implies that

$$
\begin{aligned}
& f_{G_{1}}\left(p^{-\beta}, h M_{\lambda} ; s\right) \\
& =N_{G, s-1+m / 2}\left(M_{\lambda}\right) q^{-\beta(s+m / 2)} N_{H_{1}, s-1+m / 2}(h) \frac{1-q^{-(2 s+m)}}{1-q^{-(2 s+1)}} \\
& \times\left\{1+\sum_{l=\lambda+1}^{\infty}\left(1-q^{-1}\right) q^{-2 s(l-\lambda)}\right\} \\
& =N_{G, s-1+m / 2}\left(h M_{\lambda}\right) q^{-\beta(s+m / 2)} \frac{1-q^{-(2 s+m)}}{1-q^{-2 s}} .
\end{aligned}
$$


Finally we assume that $g \in K$. Then we can take an $\eta \in L_{\mathrm{prm}}^{*}$ such that $g \in G_{\eta} K^{*}$ and $T \mid L_{\eta}$ is maximal. Replacing $M_{\lambda}$ by 1 , we can prove this case similarly.

THEOREM 2.10. Let $f$ be a right- $K^{*}$-invariant function on $G$. We assume that $f$ is a simultaneous eigenfunction of $\mathcal{H}^{+}\left(G, K^{*}\right): f * \phi=$ $\lambda_{f}(\phi) f \quad{ }^{\forall} f \in \mathcal{H}^{+}\left(G, K^{*}\right)$. Then for $g_{0} \in G$,

$$
\int_{G} f\left(g_{0} g\right) N_{G, s-1+m / 2}(g) d g=f\left(g_{0}\right) L_{\mathfrak{p}}\left(\lambda_{f} ; s\right) \prod_{j=0}^{\nu-1}\left(1-q^{-\left(2 s+n_{0}+2 j\right)}\right) B_{n_{0}}(s)
$$

where

$$
B_{n_{0}}(s)= \begin{cases}1 & n_{0}=0 \text { or } 1 \\ 1-q^{-2 s} & n_{0}=2 \\ 1-q^{-(2 s+1)} & n_{0}=3 \\ \left(1-q^{-2 s}\right)\left(1-q^{-(2 s+2)}\right) & n_{0}=4\end{cases}
$$

$\nu=\nu(S)$ is the Witt index of $S, n_{0}=n_{0}(S)=m-2 \nu$ and $L_{\mathfrak{p}}\left(\lambda_{f} ; s\right)$ is the standard L-function normalized in (1.16).

Proof. We prove our theorem by an induction on $\nu$. We set $S=S_{\nu}$ as in $(1.1)$ and denote by $(\boldsymbol{\lambda} ; \rho)=\left(\lambda_{1}, \ldots, \lambda_{\nu} ; \rho\right)$ the Satake parameter of $\lambda_{f}$. On account of Proposition 2.2 and Theorem 1.8, we have

$$
\int_{G_{\nu}} f\left(g_{0} g\right) N_{G_{\nu}, s-1+m / 2}(g) d g=f\left(g_{0}\right) \int_{G_{\nu}} \phi_{\lambda, \rho}(g) N_{G_{\nu}, s-1+m / 2}(g) d g
$$

where $\phi_{\boldsymbol{\lambda}, \rho}$ is defined in (1.11). Namely, it is sufficient to show our assertion in the case that $f=\phi_{\boldsymbol{\lambda}, \rho}$ and $g_{0}=1$. When $S$ is anisotropic (i.e. $\nu=0$ ), we have

$$
\begin{aligned}
& \int_{G_{0}} \phi_{\boldsymbol{\lambda}, \rho}(g) N_{G_{0}, s-1+n_{0} / 2}(g) d g=\sum_{u \in K_{0} / K_{0}^{*}} \rho(u) N_{G_{0}, s-1+n_{0} / 2}(u) \\
& = \begin{cases}1 & \partial=0 \\
1+\lambda_{0} q^{-\left(s-1+n_{0} / 2\right)} & \partial=1 \\
\left(1+\lambda_{0} q^{-\left(s-1+n_{0} / 2\right)}\right)\left(1+\lambda_{0} q^{-\left(s-2+n_{0} / 2\right)}\right) & \partial=2 \text { and } \lambda_{0} \neq 0 \\
1-q^{-\left(2 s-2+n_{0}\right)} & \partial=2 \text { and } \lambda_{0}=0\end{cases} \\
& =A_{\rho, \mathfrak{p}}(s) B_{n_{0}}(s) .
\end{aligned}
$$


We now assume that the theorem holds for $\nu-1$. From the definition (1.11),

$$
\phi_{\boldsymbol{\lambda}, \rho}\left(\left[\begin{array}{lll}
t & & \\
& g & \\
& & t^{-1}
\end{array}\right]\right)=|t|^{\nu-1+n_{0} / 2} \lambda_{1}(t) \phi_{\boldsymbol{\lambda}^{\prime}, \rho}(g),
$$

where we put $\lambda^{\prime}=\left(\lambda_{2}, \ldots, \lambda_{\nu}\right)$. Hence using the Iwasawa decomposition and Proposition 2.9, we get

$$
\begin{aligned}
& \int_{G_{\nu}} \phi_{\boldsymbol{\lambda}, \rho}(g) N_{G_{\nu}, s-1+m / 2}(g) d g \\
& =\int_{G_{\nu-1}} \int_{k^{\times}} \int_{k^{m-2}} \int_{K_{\nu}^{*}} \phi_{\boldsymbol{\lambda}^{\prime}, \rho}(g)|t|^{\nu-1+n_{0} / 2} \lambda_{1}(t) \\
& \quad \times N_{G_{\nu}, s-1+m / 2}\left(\left[\begin{array}{lll}
t & & \\
& g & \\
& & t^{-1}
\end{array}\right] n_{G_{\nu-1}}(X) u\right) d g d^{\times} t d X d u \\
& =\int_{G_{\nu-1}} \int_{k^{\times}} \phi_{\boldsymbol{\lambda}^{\prime}, \rho}(g) \lambda_{1}(t) q^{-\operatorname{ord}_{p} t \mid s} N_{G_{\nu-1}, s-2+m / 2}(g) \frac{1-q^{-(2 s+m-2)}}{1-q^{-2 s}} d g d^{\times} t .
\end{aligned}
$$

Therefore the inductive assumption implies that this equals

$$
\begin{aligned}
\prod_{j=1}^{\nu-1}\{(1- & \left.\left.\lambda_{j+1}(p) q^{-s}\right)\left(1-\lambda_{j+1}(p)^{-1} q^{-s}\right)\right\}^{-1} A_{\rho, \mathfrak{p}}(s) \\
& \times \prod_{j=0}^{\nu-2}\left(1-q^{-\left(2 s+n_{0}+2 j\right)}\right) B_{n_{0}}(s) \\
& \times\left\{1+\sum_{l=1}^{\infty} q^{-l s}\left(\lambda_{1}(p)^{l}+\lambda_{1}(p)^{-l}\right)\right\} \frac{1-q^{-(2 s+m-2)}}{1-q^{-2 s}} .
\end{aligned}
$$

Thus the theorem has been proved completely.

\subsection{Local Shintani functions}

We return to the situation in $\S 2.3$. Let $\Lambda$ (resp. $\lambda$ ) be a non-trivial character of $\mathcal{H}^{+}\left(H, U^{*}\right)$ (resp. $\mathcal{H}^{+}\left(G, K^{*}\right)$ ). We denote by $\operatorname{Sh}(\lambda, \Lambda)$ the set of left- $K^{*}$ and right- $U^{*}$ invariant functions $W$ on $H$ satisfying

$$
\begin{aligned}
(\phi * W * \Phi)(h) \stackrel{\text { def }}{=} & \int_{G} d x \int_{H} d y \phi(x) W\left(x h y^{-1}\right) \Phi(y) \\
= & \lambda(\phi) \Lambda(\Phi) \omega(h) \\
& { }^{\forall} \phi \in \mathcal{H}^{+}\left(G, K^{*}\right),{ }^{\forall} \Phi \in \mathcal{H}^{+}\left(H, U^{*}\right),{ }^{\forall} h \in H .
\end{aligned}
$$

We call $\operatorname{Sh}(\lambda, \Lambda)$ the space of local Shintani functions associated with $(\lambda, \Lambda)$. 
Theorem 2.11. Let $W \in \operatorname{Sh}(\lambda, \Lambda)$ and $s \in \mathbf{C}$ with sufficiently large real part. Then

$$
\begin{aligned}
Z_{W}(s) & \stackrel{\text { def }}{=} \int_{G \backslash H} W\left(\iota_{0}\left(\beta(\iota(h))^{-1}\right) h\right)|\alpha(\iota(h))|^{s+(m-1) / 2} d h \\
& =W(1) \frac{L_{\mathfrak{p}}(\Lambda ; s)}{L_{\mathfrak{p}}(\lambda ; s+1 / 2)}\left\{\begin{array}{cl}
1 & m: \text { even } \\
\zeta_{\mathfrak{p}}(2 s)^{-1} & m: \text { odd } .
\end{array}\right.
\end{aligned}
$$

Proof. We calculate the integral

$$
\int_{H} W(h) N_{H, s-1+(m+1) / 2}(h) d h
$$

in two ways. First from Therorem 2.10 for $H,(2.24)$ equals

$$
W(1) L_{\mathfrak{p}}(\Lambda ; s) \prod_{j=0}^{\nu(T)-1}\left(1-q^{-\left(2 s+n_{0}(T)+2 j\right)}\right) B_{n_{0}(T)}(s) .
$$

Secondly by using Theorem 2.8 and Theorem 2.10 for $G$, we know that (2.24) is equal to

$$
\begin{gathered}
(2.26) \int_{G \backslash H} \int_{G} W\left(\iota_{0}\left(g \beta(\iota(h))^{-1}\right) h\right) N_{G, s-1 / 2+m / 2}(g)|\alpha(\iota(h))|^{s+(m-1) / 2} d g d h \\
=L_{\mathfrak{p}}(\lambda ; s+1 / 2) \prod_{j=0}^{\nu(S)-1}\left(1-q^{-\left(2 s+1+n_{0}(S)+2 j\right)}\right) B_{n_{0}(S)}(s+1 / 2) \\
\quad \times \int_{G \backslash H} W\left(\iota_{0}\left(\beta(\iota(h))^{-1}\right) h\right)|\alpha(\iota(h))|^{s+(m-1) / 2} d h
\end{gathered}
$$

Comparing (2.25) and (2.26), we obtain our theorem.

\section{$\S 3$. Reduction of maximal symmetric matrices}

\subsection{Locally principality of lattices in a quadratic extension}

Let $k$ be an algebraic number field, $\mathfrak{o}_{k}$ the ring of integers in $k$ and $K$ a quadratic extension over $k$. We say that an $\mathfrak{o}_{k}$-submodule $L$ of $K$ is a lattice if $L$ is finitely generated and contains a $k$-basis of $K$. Moreover a lattice which forms a ring containing 1 is called an order of $K$. For a given lattice $L$, we define its order $\mathcal{O}(L)$ by

$$
\mathcal{O}(L)=\{x \in K \mid x L \subset L\} .
$$


Let $\mathcal{O}$ be an order of $K$ and denote by $\mathcal{H}(\mathcal{O})$ the set consisting of all lattices $L$ with order $\mathcal{O}$. We recall that any lattice $L \in \mathcal{H}(\mathcal{O})$ is locally principal in the following sense (cf. [2] Proposition 1, [6] Lemma 2.4).

For a place $v$ of $k, k_{v}$ means the completion of $k$ at $v$. We identify a prime ideal with the corresponding nonarchimedean place. For a prime ideal $\mathfrak{p}, \mathfrak{o}_{\mathfrak{p}}$ means the maximal order of $k_{\mathfrak{p}}$. We also put $L_{\mathfrak{p}}=L \otimes_{\mathfrak{o}_{k}} \mathfrak{o}_{\mathfrak{p}}$ and $K_{v}=K \otimes_{k} k_{v}$. Locally principality says that for any $L \in \mathcal{H}(\mathcal{O})$ and any prime ideal $\mathfrak{p}$, there exists an $\alpha_{\mathfrak{p}} \in K_{\mathfrak{p}}^{\times}$such that $L_{\mathfrak{p}}=\alpha_{\mathfrak{p}} \mathcal{O}_{\mathfrak{p}}$. For $\alpha=\left(\alpha_{v}\right) \in K_{A}^{\times}$(the idele group of $K$ ), we put

$$
\alpha \mathcal{O}=\bigcap_{\mathfrak{p}<\infty}\left(\alpha_{\mathfrak{p}} \mathcal{O}_{\mathfrak{p}} \cap K\right)
$$

Since $\alpha_{\mathfrak{p}}$ is uniquely determined modulo $\mathcal{O}_{\mathfrak{p}}^{\times}$and $L=\bigcap_{\mathfrak{p}<\infty}\left(L_{\mathfrak{p}} \cap K\right)$, we get

LEMMA 3.1. The mapping $\alpha \mapsto \alpha \mathcal{O}$ gives a bijection between $K_{A}^{\times} / K_{\infty}^{\times}$. $\prod_{\mathfrak{p}<\infty} \mathcal{O}_{\mathfrak{p}}^{\times}$and $\mathcal{H}(\mathcal{O})$. Here $K_{\infty}$ means the direct product of $K_{v}$ for all archimedean places $v$ and $\mathfrak{p}$ runs through all non-archimedean places.

For two lattices $L$ and $M$, we define its product $L \cdot M$ by the $\mathfrak{o}_{k}$-module generated by $x y(x \in L, y \in M)$. Under this multiplication, $\mathcal{H}(\mathcal{O})$ forms an abelian group and the bijection given in the above lemma becomes an isomorphism. We say that two elments $L$ and $M \in \mathcal{H}(\mathcal{O})$ are equivalent if $L=\xi M$ for some $\xi \in K^{\times}$. Let $H(\mathcal{O})$ the quotient group of $\mathcal{H}(\mathcal{O})$ by the equivalence. Then from Lemma 3.1, we obtain

$$
K_{A}^{\times} / K^{\times} K_{\infty}^{\times} \prod_{\mathfrak{p}<\infty} \mathcal{O}_{\mathfrak{p}}^{\times} \cong H(\mathcal{O}) .
$$

When $L=\alpha \mathcal{O} \in \mathcal{H}(\mathcal{O})$, we denote by $\mathfrak{n}(L)$ the (fractional) ideal of $k$ corresponding to $\mathrm{N}_{K / k}(\alpha)$. Clearly $L \mapsto \mathfrak{n}(L)$ gives a homomorphism from $\mathcal{H}(\mathcal{O})$ (resp. $H(\mathcal{O})$ ) into the ideal group of $k$ (resp. the ideal class group of $k$ ). We say that $T \in \operatorname{Sym}_{m}(k)$ represents an ideal $\mathfrak{a}$ if $\left(\frac{1}{2} T[x]\right)=\mathfrak{a}$ for some $x \in \mathfrak{o}_{k}^{m}$.

Proposition 3.2. Let $T=\left[\begin{array}{cc}2 a & b \\ b & 2 c\end{array}\right] \in \operatorname{Sym}_{2}(k)$ be non-degenerate and $\mathfrak{m}$ the ideal generated by $a, b, c$. We assume that $D=b^{2}-4 a c$ is not a square in $k$. Then there exist infinitely many primes $\mathfrak{q}$ such that $T$ represents $\mathrm{mq}$. 
Proof. We consider a quadratic extension $K=k(\sqrt{D})=k+k \omega, \omega=$ $(b-\sqrt{D}) / 2$. It is easily seen that the order of the lattice $L=\mathfrak{o}_{k} a+\mathfrak{o}_{k} \omega$ is $\mathcal{O}=\mathfrak{o}_{k}+\mathfrak{m}^{-1} \omega$. Using Lemma 3.1 we can take an $\alpha \in K_{A}^{\times}$so that $L=\alpha \mathcal{O}$. As is well-known, there exist infinitely many prime ideals $q$ which satisfy the following condition:

There exists a $\beta \in\left(\alpha^{-1} K^{\times} K_{\infty}^{\times} \prod_{\mathfrak{p}<\infty} \mathcal{O}_{\mathfrak{p}}^{\times}\right) \cap\left(K_{\infty} \prod_{\mathfrak{p}<\infty} \mathcal{O}_{\mathfrak{p}}\right)$ such that $\mathrm{N}_{K / k}(\beta)$ corresponds to $\mathfrak{q}$.

We take such $\mathfrak{q}$ and $\beta$, and put $M=\beta \mathcal{O}$. From (3.3) there exists an element $\xi \in K^{\times}$such that $M=\xi L^{-1}$. Because of $\mathfrak{n}(M)=\mathfrak{q}$ and $\mathfrak{n}(L)=(a) \mathfrak{m}$, we have $\mathfrak{n}(\xi \mathcal{O})=\left(\mathrm{N}_{K / k}(\xi)\right)=(a) \mathfrak{m q}$. Since $\xi \in M \cdot L \subset L$, we can write $\xi=a x+\omega y\left(x, y \in \mathfrak{o}_{k}\right)$ and obtain $\mathrm{N}_{K / k}(\xi)=a\left(a x^{2}+b x y+c y^{2}\right)$. This completes the proof.

\subsection{Reduction of totally positive definite maximal symmetric matrices}

Hereafter let $k$ be a totally real algebraic number field of degree $n$. We denote by $\mathcal{L}_{m}$ the set of even integral symmetric matrices of degree $m$, i.e. $\mathcal{L}_{m}=\left\{T=\left(t_{i j}\right) \in M_{m}(k) \mid t_{i j}=t_{j i} \in \mathfrak{o}_{k}, t_{i i} \in 2 \mathfrak{o}_{k}\right\}$. We say that $T \in \mathcal{L}_{m}$ is maximal if it is maximal in $\mathcal{L}_{m, \mathfrak{p}}=\mathcal{L}_{m} \otimes_{\mathfrak{o}_{k}} \mathfrak{o}_{\mathfrak{p}}$ at each prime ideal $\mathfrak{p}$ in the sense of $\S 1.1$. For a non-zero symmetric matrix $T=\left(t_{i j}\right)$ of degree $m$ with entries in $k$, we denote by $\mathfrak{m}(T)$ the (fractional) ideal in $k$ generated by $t_{i j}(i \neq j)$ and $t_{i i} / 2$.

THEOREM 3.3. Assume that $T \in \mathcal{L}_{m}$ is totally positive definite and maximal. Then there exists an element $\gamma \in S L_{m}\left(\mathfrak{o}_{k}\right)$ such that the upperleft $(m-1) \times(m-1)$ block $S$ of $T[\gamma]$ is also maximal. Moreover we can take $\gamma$ so that $\partial_{\mathfrak{p}}(S) \leq 1$ for all prime ideals $\mathfrak{p}$, where $\partial_{\mathfrak{p}}(S)$ was defined in $\S 1.1$.

The proof is given in the next subsection. We prepare a proposition.

Proposition 3.4. Let $T$ be a totally positive definite symmetric matrix of rank $m \geq 2$. Then there exist infinitely many prime ideals $\mathfrak{q}$ such that $T$ represents $\mathfrak{m}(T) \mathfrak{q}$.

Proof. We shall prove it by induction on $m$. Without loss of generality we may assume $T \in \mathcal{L}_{m}$. The case $m=2$ was proved in Proposition 3.2. Thus we assume that $m \geq 3$ and the assertion holds for $m-1$. For 
$A \in \operatorname{Sym}_{m}(k)$ and $1 \leq i \leq m, A^{(i)}$ stands for the $(m-1) \times(m-1)$ symmetric matrix obtained from $A$ by deleting the $i$-th row and $i$-th column. We take a maximal element $\mathfrak{m}_{1}$ (with respect to the inclusion) of $\left\{\mathfrak{m}\left(T[\gamma]^{(i)}\right) \mid \gamma \in\right.$ $\left.S L_{m}\left(\mathfrak{o}_{k}\right), 1 \leq i \leq m\right\}$. We may assume that $T=\left[\begin{array}{cc}T_{1} & * \\ * & *\end{array}\right]\left(T_{1} \in \mathcal{L}_{m-1}\right)$ and $\mathfrak{m}\left(T_{1}\right)=\mathfrak{m}_{1}$. From the induction hypothesis we can take an element $\gamma_{1} \in$ $S L_{m-1}\left(\mathfrak{o}_{k}\right)$ such that $T_{1}\left[\gamma_{1}\right]=\left[\begin{array}{cc}2 a & * \\ * & *\end{array}\right]$, where $\mathfrak{q}_{1}=\mathfrak{m}_{1}^{-1}(a)$ is a prime ideal not dividing $\operatorname{det} T \cdot \operatorname{det} T_{1}$. Setting $T^{\prime}=T\left[\left[\begin{array}{cc}\gamma_{1} & 0 \\ 0 & 1\end{array}\right]\right]=\left[\begin{array}{cc}2 a & { }^{t} h \\ h & T_{2}\end{array}\right]$, let us observe that each entry of $h$ is in $\mathfrak{m}_{1}$. By the strong approximation theorem for $S L_{m-1}$, there exists a $\gamma^{\prime} \in S L_{m-1}\left(\mathfrak{o}_{k}\right)$ such that ${ }^{t} h \gamma^{\prime} \equiv(c, 0, \ldots, 0)$ $\left(\bmod \mathfrak{m}_{1} \mathfrak{q}_{1}\right)$, where $c \in \mathfrak{o}_{k}$ is not in $\mathfrak{q}_{1}$. Since $\mathfrak{m}\left(T^{\prime}\left[\left[\begin{array}{cc}1 & 0 \\ 0 & \gamma^{\prime}\end{array}\right]\right]^{(m)}\right)$ is a divisor of $(a, c)$ and $(a, c)=\left(\mathfrak{m}_{1} \mathfrak{q}_{1}, c\right)=\left(\mathfrak{m}_{1}, c\right)$, the maximality of $\mathfrak{m}_{1}$ implies that $c \in \mathfrak{m}_{1}$. Now we apply our assumption to $T_{2}$. We write $\mathfrak{m}_{2}=\mathfrak{m}\left(T_{2}\right)$. Take a prime ideal $\mathfrak{q}_{2}$ not dividing $\mathfrak{q}_{1} \mathfrak{m}_{1}\left(\operatorname{det} T_{2}\right)$ and an element $\gamma_{2} \in S L_{m-1}\left(\mathfrak{o}_{k}\right)$ so that $T_{2}\left[\gamma_{2}\right]=\left[\begin{array}{cc}2 b & * \\ * & *\end{array}\right]$ and $(b)=\mathfrak{m}_{2} \mathfrak{q}_{2}$. Then we have

$$
T^{\prime \prime}=T^{\prime}\left[\left[\begin{array}{cc}
1 & 0 \\
0 & \gamma_{2}
\end{array}\right]\right]=\left[\begin{array}{ccc}
2 a & h_{12} & h_{13} \\
{ }^{t} h_{12} & 2 b & h_{23} \\
{ }^{t} h_{13} & { }^{t} h_{23} & h_{33}
\end{array}\right]
$$

where $h_{12} \in \mathfrak{m}_{1}, h_{13} \in M_{1, m-2}\left(\mathfrak{m}_{1}\right), h_{23} \in M_{1, m-2}\left(\mathfrak{m}_{2}\right)$ and $h_{33} \in \mathcal{L}_{m-2} \cdot \mathfrak{m}_{2}$. Since $\left(\mathfrak{m}_{1}, \mathfrak{m}_{2}\right)=\mathfrak{m}(T)$ and $m \geq 3$, we have $(a, b)=\left(\mathfrak{m}_{1} \mathfrak{q}_{1}, \mathfrak{m}_{2} \mathfrak{q}_{2}\right)=\mathfrak{m}(T)$. Therefore our assertion is implied by Proposition 3.2.

Remark 3.5. In the case $m=2$, Theorem 3.3 follows from Proposition 3.2 immediately.

\subsection{Proof of Theorem $\mathbf{3 . 3}$}

When $T$ is a symmetric matrix of size $m, T_{r}$ stands for the upper-left $r \times r$ block of $T(1 \leq r \leq m)$. An even integral symmetric matrix $T \in \mathcal{L}_{m, \mathfrak{p}}$ is said to be $\mathfrak{p}$-normal if it satisfies the following conditions :

$$
T_{r} \text { is maximal }(1 \leq r \leq m) \text { and } \partial_{\mathfrak{p}}\left(T_{r}\right)=0 \quad\left(1 \leq r \leq m-\partial_{\mathfrak{p}}(T)\right) \text {. }
$$

(ii-a) When $\mathfrak{p} \backslash 2, T$ is diagonal.

(ii-b) When $\mathfrak{p} \mid 2, t_{i j}=0$ for $|i-j| \geq 2$ and $t_{2 i, 2 i+1}=0$ for $2 i+1<m$. Moreover $t_{m-1, m}=0$ (resp. $t_{m-2, m-1}=0$ ) if $n_{0, \mathfrak{p}}=1$ or $\left(n_{0, \mathfrak{p}}, \partial_{\mathfrak{p}}(T)\right)=$ $(3,1)$ (resp. $\left.\left(n_{0, \mathfrak{p}}, \partial_{\mathfrak{p}}(T)\right)=(3,2)\right)$. 
We state a local version of the theorem, which is easily obtained from the Witt decomposition (1.1) and the list (1.2).

LEMMA 3.6. For any maximal $T \in \mathcal{L}_{m, \mathfrak{p}}$, there exists an element $\gamma \in$ $S L_{m}\left(\mathfrak{o}_{\mathfrak{p}}\right)$ such that $T[\gamma]$ is $\mathfrak{p}$-normal.

We return to the global situation. Let $T \in \mathcal{L}_{m}$ and let $\mathfrak{m}$ be an integral ideal. We say that $T$ is quasi-normal of level $\mathfrak{m}$ if for any prime ideal $\mathfrak{p}$ there exists a p-normal $S_{\mathfrak{p}} \in \mathcal{L}_{m, \mathfrak{p}}$ such that $T-S_{\mathfrak{p}} \in \mathfrak{m} \mathcal{L}_{m, \mathfrak{p}}$. From the strong approximation theorem for $S L_{m}$ and Lemma 3.6, we get

Lemma 3.7. Suppose that $T \in \mathcal{L}_{m}$ is maximal and $\mathfrak{m}$ is an integral ideal. Then there exists an element $\gamma \in S L_{m}\left(\mathfrak{o}_{k}\right)$ such that $T[\gamma]$ is quasinormal of level $\mathfrak{m}$.

We set

$$
\Gamma_{m}(\mathfrak{m})=\left\{\gamma \in S L_{m}\left(\mathfrak{o}_{k}\right) \mid \gamma \equiv \text { diagonal matrix }(\bmod \mathfrak{m})\right\} .
$$

It is obvious that if $T$ is quasi-normal of level $\mathfrak{m}$, so is $T[\gamma]$ for any $\gamma \in$ $\Gamma_{m}(\mathfrak{m})$. The proof of Therorem 3.3 is reduced to the following proposition.

Proposition 3.8. Suppose that $T=\left[\begin{array}{cc}T_{r} & A \\ t_{A} & S\end{array}\right] \in \mathcal{L}_{m}$ is totally positive definite, maximal and quasi-normal of level $\left(2^{s} M\right)$, where $M=(4 \operatorname{det} T)^{2}$ and $s=m-r \geq 2$. We assume that

(a) $T_{r}$ is maximal,

(b) $\partial_{\mathfrak{p}}\left(T_{r}\right)=0$ for ${ }^{\forall} \mathfrak{p} \mid M$,

(c) $\partial_{\mathfrak{p}}\left(T_{r}\right) \leq 1$ for ${ }_{\mathfrak{p}}$.

Then there exists an element $\gamma \in \Gamma_{m}\left(2^{s-1} M\right)$ such that $T^{\prime}=T[\gamma]=$ $\left[\begin{array}{cc}T_{r+1}^{\prime} & A^{\prime} \\ t^{\prime} A^{\prime} & S^{\prime}\end{array}\right]$ satisfies the following conditions.

(a') $T_{r+1}^{\prime}$ is maximal.

$\left(\mathrm{b}^{\prime}\right) \quad \partial_{\mathfrak{p}}\left(T_{r+1}^{\prime}\right) \leq \partial_{\mathfrak{p}}(T)$ for ${ }^{{ }_{\mathfrak{p}}} \mid M$. Furthermore $\partial_{\mathfrak{p}}\left(T_{r+1}^{\prime}\right)=0$ for ${ }^{{ }_{\mathfrak{p}}} \mid M$ if $s \geq 3$.

$\left(\mathrm{c}^{\prime}\right) \quad \partial_{\mathfrak{p}}\left(T_{r+1}^{\prime}\right) \leq 1$ for ${ }^{\forall} \mathfrak{p}$.

Proof. From the condition (b), any odd prime divisor of $M$ is coprime to $\operatorname{det} T_{r}$. Thus there exists a $\gamma_{1} \in \Gamma_{r}\left(2^{s} M\right)$ such that $T_{r}\left[\gamma_{1}\right]$ is quasinormal of level $\left(2^{s-1} M \operatorname{det} T_{r}\right)$. Since $T\left[\left[\begin{array}{cc}\gamma_{1} & \\ & 1\end{array}\right]\right]=\left[\begin{array}{cc}T_{r}\left[\gamma_{1}\right] & { }^{t} \gamma_{1} A \\ { }^{t} \gamma_{1} & S\end{array}\right]$ is 
quasi-normal of level $\left(2^{s} M\right)$, the $r$-th row of ${ }^{t} \gamma_{1} A$ is congruent to $(*, 0, \ldots, 0)$ modulo $2^{s} M$. We can take a $\gamma_{2} \in \Gamma_{s}\left(2^{s} M\right)$ such that the $r$-th row of ${ }^{t} \gamma_{1} A \gamma_{2}$ is congruent to $(*, 0, \ldots, 0)$ modulo $2^{s-1} M \operatorname{det} T_{r}$. We put ${ }^{t} \gamma_{1} A \gamma_{2}=$ $\left(B_{1}, B_{2}\right)\left(B_{1} \in M_{r, 1}\left(\mathfrak{o}_{k}\right), B_{2} \in M_{r, s-1}\left(\mathfrak{o}_{k}\right)\right)$. Taking into account of (a), we know $T_{r}\left[\gamma_{1}\right]^{-1} B_{2} \in M_{r, s-1}\left(\mathfrak{o}_{k}\right) 2^{s-1} M$. Therefore without loss of generality, we may assume that

$$
\left\{\begin{array}{l}
T=\left[\begin{array}{ll}
T_{r} & A \\
t^{A} & S
\end{array}\right] \text { is quasi-normal of level }\left(2^{s-1} M\right) \\
T_{r} \text { is quasi-normal of level }\left(2^{s-1} M \operatorname{det} T_{r}\right) \\
A=\left(A_{1}, 0\right), A_{1} \in M_{r, 1}\left(\mathfrak{o}_{k}\right) \\
\text { The conditions (a),(b) and (c) are satisfied. }
\end{array}\right.
$$

Now we put

$$
\left.T\left[\begin{array}{cc}
1_{r} & -T_{r}^{-1} A \\
& 1_{s}
\end{array}\right]\right]=\left[\begin{array}{cc}
T_{r} & 0 \\
0 & R
\end{array}\right] \quad\left(R=S-\left[\begin{array}{cc}
T_{r}^{-1}\left[A_{1}\right] & 0 \\
0 & 0
\end{array}\right]=\left(R_{i j}\right)\right)
$$

and calculate $\mathfrak{m}(R)$. By the definition of the $\mathfrak{p}$-normality, $\operatorname{ord}_{\mathfrak{p}} \mathfrak{m}(R)=1$ when $s=2$ and $\partial_{\mathfrak{p}}=2$. In the other cases, we get

$$
\operatorname{ord}_{\mathfrak{p}} \mathfrak{m}(R)= \begin{cases}-1 & \text { if } T_{r}^{-1}\left[A_{1}\right] \notin \mathfrak{o}_{\mathfrak{p}} \text { and } \mathfrak{p} \backslash 2 \\ -2 \operatorname{ord}_{\mathfrak{p}} 2 & \text { if } T_{r}^{-1}\left[A_{1}\right] \notin \mathfrak{o}_{\mathfrak{p}} \text { and } \mathfrak{p} \mid 2 \\ 0 & \text { otherwise. }\end{cases}
$$

Since $\left(2^{-1} \mathfrak{m}(R)^{-1} R_{11}, M\right)=\mathfrak{o}_{k}$, we have $\mathfrak{m}\left(R^{\prime}\right)=\mathfrak{m}(R)$ for $R^{\prime}=$ $R\left[\left[\begin{array}{ll}1 & \\ & 2^{s} M 1_{s-1}\end{array}\right]\right]$. From Proposition 3.4 we can take a prime ideal $\mathfrak{q}$ not dividing $M \operatorname{det} T_{r}$ and $x^{\prime} \in M_{s, 1}\left(\mathfrak{o}_{k}\right)$ so that $\left(\frac{1}{2} R^{\prime}\left[x^{\prime}\right]\right)=\mathfrak{m}(R) \mathfrak{q}$. Set $x=\left[\begin{array}{ll}1 & \\ & 2^{s} M 1_{s-1}\end{array}\right] x^{\prime}$ and take an element $X \in \Gamma_{s}\left(2^{s} M\right)$ whose first column is $x$. Finally we prove that $T^{\prime}=T\left[\left[\begin{array}{cc}1_{r} & 0 \\ 0 & X\end{array}\right]\right]$ has the required properties. The determinant of the upper-left $(r+1) \times(r+1)$ block $T_{r+1}^{\prime}$ of $T^{\prime}$ is easily calculated:

$$
\left(\operatorname{det} T_{r+1}^{\prime}\right)=\left(2 \operatorname{det} T_{r}\right) \mathfrak{q m}(R) .
$$

When $\mathfrak{p}$ divides $M,\left(\mathrm{a}^{\prime}\right)-\left(\mathrm{c}^{\prime}\right)$ are satisfied at $\mathfrak{p}$ because of the $\mathfrak{p}$-normality of $T$. On the other hand, suppose that $\mathfrak{p}$ does not divide $M$. Then $\mathfrak{p}$ is prime to 2 . By (3.7) and (3.8), we know that $\operatorname{ord}_{\mathfrak{p}} \operatorname{det} T_{r+1}^{\prime} \leq 1$. Therefore $\left(\mathbf{a}^{\prime}\right)$ and $\left(\mathbf{c}^{\prime}\right)$ hold. 


\subsection{Automorphic forms on definite orthogonal groups}

Let $S$ be an even integral totally positive definite symmetric matrix of rank $m$ and $G=O(S)$ the orthogonal group of $S$ (an algebraic group defined over $k$ ). For any prime ideal $\mathfrak{p}$, put

$$
K_{\mathfrak{p}}^{*}=\left\{k \in G L_{m}\left(\mathfrak{o}_{\mathfrak{p}}\right) \mid(k-1) S^{-1} \in M_{m}\left(\mathfrak{o}_{\mathfrak{p}}\right)\right\} .
$$

and $K_{f}^{*}=\prod_{\mathfrak{p}<\infty} K_{\mathfrak{p}}^{*}$. We denote by $G_{\infty}$ the direct product of $G_{v}$ over all archimedean places. Let $\mathfrak{S}\left(K_{f}^{*}\right)$ be the space of automorphic forms on the adele group $G_{A}$ defined to be

$$
\begin{aligned}
\mathfrak{S}\left(K_{f}^{*}\right)=\{f: & G_{A} \longrightarrow \mathbf{C} \mid f(\gamma g k)=f(g) \\
& \text { for } \left.{ }^{\forall} \gamma \in G_{k},{ }^{\forall} g \in G_{A},{ }^{\forall} k \in G_{\infty} \times K_{f}^{*}\right\} .
\end{aligned}
$$

It forms a finite dimensional vector space with the Petersson inner product

$$
<f_{1}, f_{2}>_{G}=\int_{G_{k} \backslash G_{A}} f_{1}(g) \overline{f_{2}(g)} d g,
$$

where $d g$ is the Haar measure normalized so that the total volume of $G_{\infty} \times K_{f}^{*}$ is 1 . Hereafter we assume that $S$ is maximal. The Hecke algebra $\mathcal{H}_{f}=\otimes_{\mathfrak{p}<\infty}^{\prime} \mathcal{H}\left(G_{\mathfrak{p}}, K_{\mathfrak{p}}^{*}\right)$ (restricted tensor product) acts on $\mathfrak{S}\left(K_{f}^{*}\right)$ by the convolution product. In particular the action of its center $\mathcal{H}_{f}^{+}=$ $\otimes_{p<\infty}^{\prime} \mathcal{H}^{+}\left(G_{\mathfrak{p}}, K_{\mathfrak{p}}^{*}\right)$ is normal with respect to $<,>_{G}$ (see $\S 1.2$ for the definition of $\left.\mathcal{H}^{+}\right)$. Take any $g_{0} \in G_{A}$ and write

$$
\begin{aligned}
& g_{0}=\gamma_{0} \zeta_{0} k_{0} \\
& \left(\gamma_{0} \in G L_{m}(k), \zeta_{0} \in G L_{m}\left(k_{\infty}\right), k_{0}=\prod_{\mathfrak{p}<\infty} k_{0, \mathfrak{p}}, k_{0, \mathfrak{p}} \in G L_{m}\left(\mathfrak{o}_{\mathfrak{p}}\right)\right) .
\end{aligned}
$$

Then clearly $S^{\prime}=S\left[\gamma_{0}\right]$ is also a maximal even integral symmmetric matrix. We define $G^{\prime}=O\left(S^{\prime}\right), K_{\mathfrak{p}}^{\prime *}$ and $K_{f}^{\prime *}$ similarly. For any $f \in \mathfrak{S}\left(K_{f}^{*}\right)$ (resp. $\phi \in \mathcal{H}\left(G_{\mathfrak{p}}, K_{\mathfrak{p}}^{*}\right)$ ), we define a function $f^{\prime}$ on $G_{A}^{\prime}$ (resp. $\phi^{\prime}$ on $G_{\mathfrak{p}}^{\prime}$ ) by

$$
\begin{aligned}
& f^{\prime}\left(g^{\prime}\right)=f\left(\gamma_{0} g^{\prime} \gamma_{0}^{-1} g_{0}\right) \quad\left(g^{\prime} \in G_{A}^{\prime}\right) \\
& \phi^{\prime}\left(g^{\prime}\right)=\phi\left(k_{0, \mathfrak{p}}^{-1} g^{\prime} k_{0, \mathfrak{p}}\right) \quad\left(g^{\prime} \in G_{\mathfrak{p}}^{\prime}\right) .
\end{aligned}
$$

Proposition 3.9. Let notation and assumption be as above.

(i) The mapping $f \mapsto f^{\prime}$ (resp. $\left.\phi \mapsto \phi^{\prime}\right)$ gives an isomorphism between $\mathfrak{S}\left(K_{f}^{*}\right)$ and $\mathfrak{S}\left(K_{f}^{\prime *}\right)\left(\right.$ resp. $\mathcal{H}\left(G_{\mathfrak{p}}, K_{\mathfrak{p}}^{*}\right)$ and $\left.\mathcal{H}\left(G_{\mathfrak{p}}^{\prime}, K_{\mathfrak{p}}^{\prime *}\right)\right)$.

(ii) Both isomorphisms are compatible with respect to the actions of Hecke algebras, namely for any $f \in \mathfrak{S}\left(K_{f}^{*}\right)$ and $\phi \in \mathcal{H}\left(G_{\mathfrak{p}}, K_{\mathfrak{p}}^{*}\right)$ the relation $f^{\prime} *$ $\phi^{\prime}=(f * \phi)^{\prime}$ holds. 
Proof. The first part of the proposition is trivial. We check here the second part. For any $x^{\prime} \in G_{A}^{\prime}$, we have

$$
\begin{aligned}
\left(f^{\prime} * \phi^{\prime}\right)\left(x^{\prime}\right) & =\int_{G_{\mathfrak{p}}^{\prime}} f^{\prime}\left(x^{\prime} y^{\prime}\right) \phi^{\prime}\left(y^{\prime-1}\right) d y^{\prime} \\
& =\int_{G_{\mathfrak{p}}} f\left(\gamma_{0} x^{\prime} k_{0, \mathfrak{p}} y k_{0, \mathfrak{p}}^{-1} \gamma_{0}^{-1} g_{0}\right) \phi\left(y^{-1}\right) d y \\
& =(f * \phi)\left(\gamma_{0} x^{\prime} \gamma_{0}^{-1} g_{0}\right) .
\end{aligned}
$$

Thus we obtain (ii).

Remark 3.10. Under this correspondence, $f\left(g_{0}\right) \neq 0$ if and only if $f^{\prime}(1) \neq 0$. Therefore to study the $L$-function associated with $f$, we may assume that the upper-left $(m-1) \times(m-1)$ block of $S$ is also maximal and $f(1) \neq 0$.

\section{$\S 4$. Functional equation of the standard $L$-function}

\subsection{Main theorem}

As in the previous section, let $k$ be a totally real algebraic number field of degree $n$ and $S \in \mathcal{L}_{m}$ a totally positive definite, maximal even integral symmetric matrix of rank $m$. We denote by $G$ the orthogonal group of $S$. As in $\S 4.3$, we consider the space $\mathfrak{S}\left(K_{f}^{*}\right)$ of automorphic forms on $G_{A}$ with respect to $K_{f}^{*}=\prod_{\mathfrak{p}<\infty} K_{\mathfrak{p}}^{*}\left(K_{\mathfrak{p}}^{*}\right.$ is defined in (3.9)). Let $f \in \mathfrak{S}\left(K_{f}^{*}\right)$ be a simultaneous eigenfunction of $\mathcal{H}_{f}^{+}=\otimes_{\mathfrak{p}<\infty}^{\prime} \mathcal{H}^{+}\left(G_{\mathfrak{p}}, K_{\mathfrak{p}}^{*}\right)$ :

$$
f * \phi=\lambda_{f, \mathfrak{p}}(\phi) f \quad{ }^{\forall} \phi \in \mathcal{H}^{+}\left(G_{\mathfrak{p}}, K_{\mathfrak{p}}^{*}\right) .
$$

Then we define the (global) standard $L$-function attached to $f$ by

$$
L(f ; s)=\prod_{\mathfrak{p}<\infty} L_{\mathfrak{p}}\left(\lambda_{f, \mathfrak{p}} ; s\right) \quad(s \in \mathbf{C}),
$$

where $L_{\mathfrak{p}}\left(\lambda_{f, \mathfrak{p}} ; s\right)$ is the local standard $L$-function normalized in (1.16). As the gamma factor, we take

$$
\begin{aligned}
L_{\infty}(f ; s)=\left|d_{k}\right|^{[m / 2] s} & \left((2 \pi)^{-[m / 2] s} \prod_{j=1}^{[m / 2]} \Gamma(s-j+m / 2)\right)^{n} \\
& \times \begin{cases}\left(\mathrm{N}_{k / \mathbf{Q}}(\operatorname{det} S)\right)^{s / 2} & m: \text { even } \\
\left(\mathrm{N}_{k / \mathbf{Q}}\left(2^{-1} \operatorname{det} S\right)\right)^{s / 2} & m: \text { odd }\end{cases}
\end{aligned}
$$

where $d_{k}$ is the discriminant of $k$.

Now we state our main theorem of the paper. 
THEOREM 4.1. Let $f \in \mathfrak{S}\left(K_{f}^{*}\right)$ be a simultaneous eigenfunction of $\mathcal{H}_{f}^{+}$. (i) The function $\xi(f ; s)=L_{\infty}(f ; s) L(f ; s)$ is continued to $\mathbf{C}$ as a meromorphic function of $s$ and satisfies the functional equation $\xi(f ; s)=\xi(f ; 1-s)$. (ii) When $m=1, \xi(f ; s)$ is entire. It does not vanish at $s=1 / 2$ if and only if $f$ is a constant function.

(iii) When $m \geq 2, \xi(f ; s)$ is holomorphic except for possible simple poles at $s=m / 2-k \quad(0 \leq k \leq m-1, k \in \mathbf{Z})$. It has a simple pole at $s=m / 2$ if and only if $f$ is a constant function.

Remark 4.2. Our definition of the gamma factor is slightly different from that in [4] $\S 1$.

To prove the theorem, we adopt the same strategy as in [4], where the assertion was partially proved. First we check the theorem for $m=$ 1. From the maximality condition, the ideal $\left(\frac{1}{2} S\right)$ is a product $\mathfrak{p}_{1} \cdots \mathfrak{p}_{r}$ of distinct prime ideals $\mathfrak{p}_{i}$. Thus for a simultaneous eigenfunction $f \in$ $\mathfrak{S}\left(K_{f}^{*}\right), \xi(f ; s)=\mathrm{N}_{k / \mathbf{Q}}(S / 2)^{s / 2} \prod_{j=1}^{r}\left(1+\lambda_{f, \mathfrak{p}_{j}, 0} \mathrm{~N}_{k / \mathbf{Q}}\left(\mathfrak{p}_{j}\right)^{-(s-1 / 2)}\right)$, where $\lambda_{f, \mathfrak{p}_{j}, 0}(= \pm 1)$ is determined by the action of $-1 \in G_{\mathfrak{p}_{j}}$. Clearly $\xi(f ; s)$ is entire. Noting that $-1 \in G_{k}$ is embedded into $G_{A}$ diagonally and hence that $\prod_{j=1}^{r} \lambda_{f, \mathfrak{p}_{j}, 0}=1$, we get the functional equation $\xi(f ; s)=\xi(f ; 1-s)$. Moreover since $f$ is constant on $G_{A}$ if and only if $\lambda_{f, \mathfrak{p}_{j}, 0}=1$ for all $j$, the characterization of constant functions is obtained.

Hereafter we assume that our theorem holds for $m$ and let us prove it for $m+1$.

\subsection{Eisenstein series}

To describe the basic identity in the next subsection, we introduce an Eisenstein series. Let $G_{1}$ be the orthogonal group of $S_{1}=\left[\begin{array}{lll} & S & 1 \\ 1 & & \end{array}\right]$. For any prime ideal $\mathfrak{p}$, put $K_{1, \mathfrak{p}}^{*}=\left\{k_{1} \in G_{1, \mathfrak{p}} \mid\left(k_{1}-1\right) S_{1}^{-1} \in M_{m+2}\left(\mathfrak{o}_{\mathfrak{p}}\right)\right\}$. Let $v_{1}, \ldots, v_{n}$ be the archimedean places of $k$. We recall the action of $G_{1, v_{i}}$ on $\mathcal{D}=\mathbf{R}^{m} \times \mathbf{R}_{+}^{\times}\left(\mathbf{R}_{+}^{\times}\right.$is the set of positive real numbers $)$. For $X=(x, r) \in \mathcal{D}$, put $X^{\sim}=\left[\begin{array}{c}-r-S[x] / 2 \\ x \\ 1\end{array}\right] \in \mathbf{R}^{m+2}$. We define the action $g\left\langle X>\right.$ of $G_{1, v_{i}}$ on $\mathcal{D}$ and the automorphy factor $j(g, X) \in \mathbf{R}^{\times}$on $G_{1, v_{i}} \times \mathcal{D}$ by

$$
g \cdot X^{\sim}=(g<X>)^{\sim} \cdot j(g, X) \quad\left(g \in G_{1, v_{i}}, X \in \mathcal{D}\right)
$$


We fix a point $X_{0, v_{i}}=\left(x_{0, v_{i}}, r_{0, v_{i}}\right) \in \mathcal{D}$ and denote by $K_{1, v_{i}}^{*}$ the stabilizer subgroup of $X_{0, v_{i}}$ in $G_{1, v_{i}}$. Clearly $K_{1, v_{i}}^{*}$ is a maximal compact subgroup of $G_{1, v_{i}}$ and $G_{1, v_{i}} / K_{1, v_{i}}^{*} \cong \mathcal{D}$.

Let $P_{1}$ be a maximal parabolic subgroup of $G_{1}$ defined by

$$
P_{1, k}=\left\{\left[\begin{array}{ccc}
\alpha & * & * \\
& \beta & \alpha^{*}
\end{array}\right] \mid \alpha \in k^{\times}, \beta \in G_{k}\right\} .
$$

By the Iwasawa decomposition for $G_{1, A}$, each $g \in G_{1, A}$ is written in the form

$$
g=\left[\begin{array}{ccc}
\alpha(g) & * & * \\
& \beta(g) & * \\
& & \alpha(g)^{-1}
\end{array}\right] k(g),
$$

where $\alpha(g) \in k_{A}^{\times}, \beta(g) \in G_{A}$ and $k(g) \in \prod_{v \leq \infty} K_{1, v}^{*}$. For $f \in \mathfrak{S}\left(K_{f}^{*}\right)$ and $s \in \mathbf{C}$, we define a function $f(g ; s)$ on $G_{1, A}$ by

$$
f(g ; s)=f(\beta(g))|\alpha(g)|_{A}^{s},
$$

where $|\alpha|_{A}$ means the idele norm of $\alpha \in k_{A}^{\times}$. Then the Eisenstein series associated with $f$ is defined by

$$
E(g, f ; s)=\sum_{\gamma \in P_{1, k} \backslash G_{1, k}} f\left(\gamma g ; s+\frac{m}{2}\right),
$$

which converges absolutely in a right half plane $\{s \in \mathbf{C} \mid \operatorname{Re} s>m / 2\}$. When $f$ is a simultaneous eigenfunction of $\mathcal{H}_{f}^{+}$, we introduce the normalized Eisenstein series by

$$
E^{*}(g, f ; s)=r_{0}^{s / 2} \xi(f ; s+1) E(g, f ; s) \times\left\{\begin{array}{cl}
1 & m: \text { even } \\
\xi_{k}(2 s+1) & m: \text { odd }
\end{array},\right.
$$

where $\xi_{k}(s)=\left|d_{k}\right|^{s / 2}\left(\pi^{-s / 2} \Gamma(s / 2)\right)^{n} \zeta_{k}(s)$ is the completed Dedekind zeta function of $k$ and $r_{0}=\prod_{i=1}^{n} r_{0, v_{i}}$.

Proposition 4.3. Let $f \in \mathfrak{S}\left(K_{f}^{*}\right)$ be a simultaneous eigenfunction of $\mathcal{H}_{f}^{+}$. We assume that Theorem 4.1 is valid for $f$. Then the normalized Eisenstein series $E^{*}(g, f ; s)$ has an analytic continuation to $\mathbf{C}$ as a meromorphic function of $s$ and satisfies the functional equation $E^{*}(g, f ; s)=$ $E^{*}(g, f ;-s)$. Furthermore, it is holomorphic except for possible simple poles at $s=m / 2-k(0 \leq k \leq m, k \in \mathbf{Z})$ and the residue at $s=m / 2$ is given by

$$
\operatorname{Res}_{s=m / 2} E^{*}(g, f ; s)=r_{0}^{-m / 4} f(1) \times \begin{cases}\operatorname{Res}_{s=m / 2} \xi(f ; s) & m: \text { even } \\ \underset{s=m / 2}{\operatorname{Res}} \xi(f ; s) \xi(2 s) & m: \text { odd }\end{cases}
$$


Proof. It is enough to calculate the constant term of Eisenstein series:

$$
\begin{aligned}
E_{0}(g, f ; s) & =\int_{N_{1, k} \backslash N_{1, A}} E(n g, f ; s) d n \\
& =f(g ; s+m / 2)+\int_{N_{1, A}} f(w n g ; s+m / 2) d n
\end{aligned}
$$

where $N_{1}$ is the unipotent radical of $P_{1}$ and $w=\left[\begin{array}{lll} & & 1 \\ 1 & & \end{array}\right]$. From Corollary 1.10 , we obtain

$$
\begin{aligned}
& E_{0}\left(\left[\begin{array}{lll}
\alpha_{0} & & \\
& \beta_{0} & \\
& & \alpha_{0}^{-1}
\end{array}\right], f ; s\right) \\
& =f\left(\beta_{0}\right)\left|\alpha_{0}\right|_{A}^{s+m / 2}+\frac{L(f ; s)}{L(f ; s+1)}\left\{\begin{array}{cc}
1 & m: \text { even } \\
\frac{\zeta_{k}(2 s)}{\zeta_{k}(2 s+1)} & m: \text { odd }
\end{array}\right\} \\
& \quad \times\left|d_{k}\right|^{-m / 2}\left|\alpha_{0, f}\right|_{A}^{-s+m / 2} f\left(\beta_{0}\right) \\
& \quad \times \prod_{i=1}^{n} \int_{\mathbf{R}^{m}}\left|\alpha\left(w\left[\begin{array}{ccc}
1 & -{ }^{t} x S & -S[x] / 2 \\
1_{m} & \frac{x}{1}
\end{array}\right]\left[\begin{array}{ccc}
\alpha_{0, v_{i}} & \beta_{0, v_{i}} & \\
& \alpha_{0, v_{i}}^{-1}
\end{array}\right]\right)\right|^{s+m / 2} d x,
\end{aligned}
$$

where $\alpha_{0, f}\left(\operatorname{resp} . \alpha_{0, v_{i}}\right)$ means the finite part (resp. the $v_{i}$ part) of $\alpha_{0}$. We can easily see that the last integral is equal to

$$
r_{0, v_{i}}^{-s}\left|\alpha_{0, v_{i}}\right|^{-s+m / 2}|\operatorname{det} S|_{v_{i}}^{-1 / 2} \frac{(2 \pi)^{m / 2} \Gamma(s)}{\Gamma(s+m / 2)} .
$$

Therefore we know that the constant term of $E^{*}(g, f ; s)$ is

$$
\begin{aligned}
& r_{0}^{s / 2} \xi(f ; s+1)\left\{\begin{array}{cc}
1 & m: \text { even } \\
\xi_{k}(2 s+1) & m: \text { odd }
\end{array}\right\} f(g ; s+m / 2) \\
& +r_{0}^{-s / 2} \xi(f ; s)\left\{\begin{array}{cc}
1 & m: \text { even } \\
\xi_{k}(2 s) & m: \text { odd }
\end{array}\right\} f(g ;-s+m / 2) .
\end{aligned}
$$

From the assumption for $f$, we obtain the proposition.

\subsection{Basic identity and the end of proof}

Let $T=\left[\begin{array}{cc}S & -S \alpha \\ -{ }^{t} \alpha S & -2 a\end{array}\right] \in \mathcal{L}_{m+1}$ be maximal and totally positive definite. We denote by $H$ the orthogonal group of $T$ and put $U_{\mathfrak{p}}^{*}=\{u \in$ 
$\left.H_{\mathfrak{p}} \mid(u-1) T^{-1} \in M_{m+1}\left(\mathfrak{o}_{\mathfrak{p}}\right)\right\}, U_{f}^{*}=\prod_{\mathfrak{p}<\infty} U_{\mathfrak{p}}^{*}$. Let $F \in \mathfrak{S}\left(U_{f}^{*}\right)$ be a simultaneous eigenfunction of $\otimes_{\mathfrak{p}<\infty}^{\prime} \mathcal{H}^{+}\left(H_{\mathfrak{p}}, U_{\mathfrak{p}}^{*}\right)$. We shall prove the assertion in Theorem 4.1 for $F$. In view of Remark 3.10, we may assume that $F(1) \neq 0$ and $S$ is also maximal. Let us embed $G=O(S)$ into $H=O(T)$ and $H$ into $G_{1}$, similarly as in $\S 2.3$. For each archimedean place $v$, we take $(\alpha,-\Delta / 2)\left(\Delta=S_{1}[\eta]=2 a+S[\alpha]\right)$ as the origin $X_{0, v} \in \mathcal{D}$. Then $\iota\left(U_{\mathfrak{p}}^{*}\right)=\iota\left(H_{\mathfrak{p}}\right) \cap K_{1, \mathfrak{p}}^{*}, \iota\left(H_{v}\right) \subset K_{1, v}^{*}$ and $j\left(\iota(h), X_{0, v}\right)=1$ for any $h \in H_{v}$. Take any simultaneous eigenfunction $f \in \mathfrak{S}\left(K_{f}^{*}\right)$ and introduce the (global) Shintani function $W_{F, f}$ associated with $F$ and $f$ by

$$
W_{F, f}(h)=\int_{G_{k} \backslash G_{A}} F\left(\iota_{0}(g) h\right) f(g) d g .
$$

We can prove the next theorem is proved quite similarly as in $[4$, Theorem $1.5]$.

Theorem 4.4. (Basic Identity)

$$
\begin{aligned}
Z_{F, f}(s) & \stackrel{\text { def }}{=} \int_{H_{k} \backslash H_{A}} F(h) E(\iota(h), f ; s-1 / 2) d h \\
& =\int_{G_{A} \backslash H_{A}} W_{F, f}\left(\iota_{0}\left(\beta(\iota(h))^{-1}\right) h\right)|\alpha(\iota(h))|_{A}^{s+(m-1) / 2} d h .
\end{aligned}
$$

By using Theorem 2.11, we know that

$$
\int_{H_{k} \backslash H_{A}} F(h) E^{*}(\iota(h), f ; s-1 / 2) d h=c \xi(F ; s) W_{F, f}(1) \quad(c \neq 0) .
$$

Since $F(1) \neq 0$, we can take a simultaneous eigenfunction $f \in \mathfrak{S}\left(K_{f}^{*}\right)$ so that $W_{F, f}(1)=\left\langle\left. F\right|_{G_{A}}, \bar{f}\right\rangle_{G} \neq 0$. Therefore combining Proposition 4.3 and (4.11), we obtain the meromorphic continuation, location of possible poles and the invariance of $\xi(f ; s)$ under $s \mapsto 1-s$. Noting that $\langle F, \mathbf{1}\rangle_{H} \neq 0$ ( 1 means the constantly 1 function) if and only if $F$ is a constant function, we get the characterization of the holomorphy of $\xi(F ; s)$ at $s=(m+1) / 2$. We have proved Theorem 4.1 completely.

\section{REFERENCES}

[1] Eichler, M., Quadratische Formen und Orthogonale Gruppen, Springer, 1952. 
[2] Ihara, Y., On certain arithmetical Dirichlet series, J. Math. Soc. Japan, 16 (1964), 214-225.

[3] Langlands, R.P., Euler product, Yale Univ. Press, 1971.

[4] Murase, A. and Sugano, T., Shintani function and its application to automorphic L-functions for classical groups, I. The case of orthogonal groups, Math. Ann., 299 (1994), 17-56.

[5] Satake, I., Theory of spherical functions on reductive algebraic groups over $\mathfrak{p}$-adic fields, Publ. Math. I.H.E.S., 18 (1963), 5-69.

[6] Sugano, T., On holomorphic cusp forms on quaternion unitary groups of degree 2, J. Fac. Sci. Univ. Tokyo, 31 (1985), 521-568..

[7] Tamagawa, T., On Selberg's trace formula, J. Fac. Sci. Univ. Tokyo, 8 (1960), 363-386.

\author{
Atsushi Murase \\ Department of Mathematics \\ Faculty of Science \\ Kyoto Sangyo University \\ Motoyama, Kamigamo \\ Kita-ku, Kyoto 603-8555, Japan \\ murase@cc.kyoto-su.ac.jp \\ Takashi Sugano \\ Department of Mathematics \\ Faculty of Science \\ Kanazawa University \\ Kakuma-machi, Kanazawa 920-1192, Japan \\ sugano@kappa.s.kanazawa-u.ac.jp
}

